\title{
MAGI-1 is a component of the glomerular slit diaphragm that is tightly associated with nephrin
}

\author{
Susumu Hirabayashi ${ }^{1}$, Hiroki Mori ${ }^{1}$, Ai Kansaku ${ }^{1}$, Hidetake Kurihara ${ }^{2}$, Tatsuo Sakai ${ }^{2}$, \\ Fujio Shimizu ${ }^{3}$, Hiroshi Kawachi ${ }^{3}$ and Yutaka Hata ${ }^{1}$ \\ ${ }^{1}$ Department of Medical Biochemistry, Graduate School of Medicine, Tokyo Medical and Dental University, \\ Tokyo, Japan; ${ }^{2}$ Department of Anatomy, Juntendo University School of Medicine, Tokyo, Japan and \\ ${ }^{3}$ Department of Cell Biology, Institute of Nephrology, Niigata University Graduate School of Medical \\ and Dental Sciences, Niigata, Japan
}

\begin{abstract}
MAGUK with inverted domain structure-1 (MAGI-1) is a membrane-associated protein with one guanylate kinase, six PSD-95/DIg-A/ZO-1 (PDZ), and two WW domains and is localized at tight junctions in epithelial cells. MAGI-1 interacts with various proteins and is proposed to function as a scaffold protein. In the previous study, we discovered a MAGI-1-interacting cell adhesion molecule junctional adhesion molecule 4 (JAM4). Both proteins are highly expressed in glomerular podocytes in the kidney and partially colocalized. In this study, we have further searched for a binding partner of MAGI-1 in the kidney through yeast two-hybrid screening and obtained nephrin. Nephrin is a cell adhesion molecule specifically localized at the slit diaphragm between neighboring foot processes of podocytes. Biochemical studies reveal that nephrin directly binds to the middle PDZ domains of MAGI-1 through its carboxyl terminus but does not bind to ZO-1. MAGI-1 forms a tripartite complex with nephrin and JAM4 in vitro. Immunoelectron microscopy shows that the localization of MAGI-1 is restricted to the slit diaphragm, whereas JAM4 is also distributed on apical membranes of podocytes. In puromycin aminonucleoside-induced nephrotic podocytes, MAGI-1 is localized with nephrin at the displaced slit diaphragm. These data indicate that MAGI-1 is a component of the slit diaphragm and tightly interacts with nephrin and JAM4 in vivo. MAGI-1 may play a role in determining the boundary between the apical and the bosolateral domain at the level of slit diaphragm.
\end{abstract}

Laboratory Investigation (2005) 85, 1528-1543. doi:10.1038/labinvest.3700347; published online 12 September 2005

Keywords: cell adhesion; cytoskeleton; PDZ; podocyte; nephrosis; scaffold

Podocytes are highly specialized cells responsible for maintaining the filtration barrier of the kidney glomerulus. Podocytes consist of a large cell body, major processes and foot processes with their interconnecting specialized cell-cell contacts called the slit diaphragm. The slit diaphragm forms the final filtration barrier that prevents serum proteins from leaking into the urine from the glomerular capillary. Thereby, the analysis of molecular nature

Correspondence: Dr H Kurihara, PhD, Department of Anatomy, Juntendo University School of Medicine, Tokyo 113-8421, Japan and $\mathrm{Dr}$ Y Hata, PhD, Department of Medical Biochemistry, Graduate School of Medicine, Tokyo Medical and Dental University, Tokyo 113-8519, Japan.

E-mails: hidetake@med.juntendo.ac.jp,

yuhammch@med.tmd.ac.jp

Received 20 June 2005; revised 4 August 2005; accepted 8 August 2005; published online 12 September 2005 of the slit diaphragm is important to understand the pathogenesis of proteinuria.

Nephrin is a transmembrane component of the slit diaphragm. ${ }^{1}$ NPHS1, the gene encoding for nephrin, is mutated in the congenital nephrotic syndrome of the Finnish type. ${ }^{2}$ Nephrin is a cell adhesion molecule of the immunoglobulin (Ig) superfamily with eight Ig-like domains and a fibronectin type III repeat in its extracellular region and is suggested to participate in forming the slit barrier via homophilic interaction. ${ }^{2,3}$ Podocin is encoded by NPHS2, whose mutation causes a steroid-resistant focal and segmental glomerulosclerosis (FSGS). ${ }^{4}$ Podocin is a hairpin-like integral membrane protein and is associated with a raft at the slit diaphragm. ${ }^{5}$ CD2associated protein (CD2AP) is an adaptor protein with three SH3 domains, a proline-rich sequence and a coiled-coil domain. ${ }^{6} \mathrm{CD} 2 \mathrm{AP}$ was first reported as a protein interacting with CD2 in T cells and is 
implicated in the regulation of receptor tyrosine kinases and TGF signaling. ${ }^{6}$ CD2AP is localized at the cytoplasmic face of the slit diaphragm and interacts with the actin cytoskeleton. ${ }^{7,8}$ Mice lacking CD2AP develop nephrotic syndrome with effacement of podocyte foot processes. ${ }^{9}$ Mutations in CD2AP gene have been reported in FSGS patients. ${ }^{10}$ Neph1 was identified as a protein homologous to nephrin. ${ }^{11}$ Neph1 shows high expression in the kidney and is colocalized with nephrin at the podocyte foot process. ${ }^{12,13}$ The deletion of Neph1 in the mice results in proteinuria. ${ }^{11} \alpha$-Actinin-4, an actin-binding and crosslinking protein, is highly expressed in podocytes. ${ }^{14}$ Mutations of $\alpha$-actinin-4 gene also cause familial FSGS. ${ }^{14}$ These proteins interact with each other. Podocin interacts with CD2AP, nephrin, and Neph1. ${ }^{5,15,16}$ The disruption of podocin is associated with abnormal distribution of nephrin and CD2AP. ${ }^{17}$ Nephrin and Neph1 form heterooligomers. ${ }^{12}$ The inhibition of the nephrinNeph1 interaction by antibodies brings in slit diaphragm dysfunction. ${ }^{13}$ These reports support the conclusion that intimate interactions among slit diaphragm proteins and slit diaphragm-associated proteins are important for the glomerular filtration barrier.

Zonula occludens-1 (ZO-1), a well-known membrane-associated guanylate kinase (MAGUK), has three PSD-95/Dlg-A/ZO-1 (PDZ) domains and binds claudins at tight junctions in epithelial cells. ${ }^{18,19} \mathrm{In}$ podocytes, ZO-1 is localized at the slit diaphragm and interacts with Neph1. ${ }^{13,19-21}$ MAGUK with inverted domain structure-1 (MAGI-1) is a tight junction-associated protein composed of one guanylate kinase, two WW, and six PDZ domains. ${ }^{22,23}$ In kidneys, MAGI-1 is enriched in glomerular podocytes. $^{24,25}$ MAGI-1 interacts with $\alpha$-actinin-4 and synaptopodin in podocytes and is linked to the actin cytoskeleton through these proteins. ${ }^{26}$ These findings suggest that MAGI-1 is involved in the molecular architecture of the slit diaphragm. We previously reported junctional adhesion molecule 4 (JAM4), a cell adhesion molecule with two Ig-like domains that binds MAGI- $1 .{ }^{25}$ JAM4 is expressed in podocytes and detected at the slit diaphragm. ${ }^{25}$ However, the localization of JAM4 does not completely overlap with MAGI-1 in various tissues. ${ }^{27}$

In this study, to further clarify the contribution of MAGI-1 to the slit diaphragm, we searched for a MAGI-1-binding protein through yeast two-hybrid screening. We found that nephrin binds MAGI-1. We extended our previous report that MAGI-1 is localized at the slit diaphragm in normal kidney and examined the localization of MAGI-1 in developing kidney and in podocytes of the proteinuric state. The data indicate that MAGI-1 is tightly associated with the slit diaphragm. CD2AP and podocin are reported to be dissociated from the displaced slit diaphragm and nephrin in nephrotic state. ${ }^{28,29}$ However, MAGI-1 remains attached to the slit diaphragm and bound to nephrin in puromycin aminonucleoside (PAN)-induced nephrosis. Thereby, the interaction between MAGI-1 and nephrin is very stable and may be a core for the assembly of the slit diaphragm.

\section{Materials and methods}

\section{Construction of Expression Vectors}

Polymerase chain reaction (PCR) was performed by using primers $\left(5^{\prime}\right.$-aggcctaatgagtgaggtaactc- $3^{\prime}$ and $5^{\prime}$-gacgtcgttcaggtggcacttttc-3') on pGBKT7 (BD Biosciences Clontech) and the PCR fragment was digested by StuI/AatII and ligated into PvuII/AatII sites of pBTM116 to generate pBTM116 KM vector. pCIneoMyc MAGI-1-I, -II, -III, -IV, -V, pCIneoMyc ZO-1-1, pFLAG-JAM4, and pGex4T-1 JAM4-C were described previously. ${ }^{25,30,31}$ Human cDNA of nephrin was obtained by PCR using primers (5'-aagcttttggcgattcctgcctccgtt- $3^{\prime}$ and $5^{\prime}$-gtcgactacaccagatgtcccctcagctc- $3^{\prime}$ ) on human kidney cDNA (BioChain, Inc.). The following constructs were made using pGex4T-1 (Amersham Pharmacia Biotech) and pFLAG-CMV-1 (Eastman Kodak Company) and encoded the corresponding amino acids of human nephrin or human MAGI-1: pFLAG nephrin, amino acids 24-1241; pFLAG nephrin-del C, 24-1236; pGex4T-1 nephrin-C, 1087-1241 of nephrin; pGex4T-1 nephrin-C del, 1087-1236; pBTM116 KM MAGI-1-8, 430-1256; pGex4T-1 MAGI-1-A, 565-994; pGex4T-1 MAGI-1-B, 565-836; pGex4T-1 MAGI-1-C, 771-994; pGex4T-1 MAGI-1-D, 565-865; pGex4T-1 MAGI-1-E, 697-994; pGex4T-1 MAGI-1-F, 697-865. Throughout this study, we use the term MAGI-1 to describe all constructs that are derived from human brain angiogenesis inhibitor protein 1-associated protein 1.

\section{Antibodies}

Antibodies against MAGI-1 and JAM4 were described previously. ${ }^{25}$ The rabbit antibody against the product of WW domains of S-SCAM (a neuronal isoform of MAGI-1, also called MAGI-2) was used for the immunoprecipitation of MAGI-1. For immunohistochemistry and immunoelectron microscopy, the affinity-purified rabbit antibody against the product of pGex S-SCAM-7 was used to detect MAGI-1. The rabbit anti-JAM4 antibody was raised against the product of pGex4T-1 JAM4-C. Affinitypurified rabbit anti-nephrin and mouse anti-nephrin antibodies (5-1-6) were described previously. ${ }^{32,33}$ Mouse anti-nephrin antibodiy 5-1-6 that reacts with the extracellular domain of rat nephrin was used for the immunofluorescence and immunogold studies. ${ }^{34}$ Mouse monoclonal anti-podocalyxin antibody (5A) was a gift from Dr Farquhar (University of California, San Diego, CA, USA). ${ }^{35}$ Mouse monoclonal anti-ZO-1 antibody was from Zymed 
Laboratories Inc. Mouse anti-Myc-tag monoclonal antibody 9E10 was obtained from the American Type Culture Collection. Mouse anti-FLAG antibody was purchased from Sigma-Aldrich. Rat anti-HA antibody was purchased from Roche Molecular Biochemicals.

\section{Yeast Two-Hybrid Screening}

Yeast two-hybrid screening was performed using a human kidney cDNA library (BD Biosciences Clontech) and yeast strain L40. We used a bait vector with kanamycin resistance gene to facilitate the recovery of candidate clones from pACT2 library. Histidine selection plates contained $8 \mathrm{mM} 3$-amino1,2,4-triazole and $360 \mathrm{mg} / \mathrm{l}$ of 5-bromo-4-chloro3 -indolyl- $\beta$-D-galactopyranoside. After 6 days of incubation, blue colonies were picked up for further analysis.

\section{Cell Culture}

COS-7 cells were grown in cultures in Dulbecco's modified Eagle's medium supplemented with $10 \%$ fetal bovine serum, $100 \mathrm{U}$ of penicillin $/ \mathrm{ml}$, and $100 \mu \mathrm{g}$ of streptomycin $/ \mathrm{ml}$ under $5 \% \mathrm{CO}_{2}$ at $37^{\circ} \mathrm{C}$. COS-7 cells were transfected with DEAE-dextran method.

\section{Immunoprecipitation from Heterologous Cells}

COS-7 cells were cotransfected by DEAE-dextran with pClneo Myc MAGI-1 and either pFLAG nephrin or pFLAG nephrin-del C. Cells from two 10-cm diameter plates were homogenized in $400 \mu \mathrm{l}$ of $25 \mathrm{mM}$ Tris-HCl (pH 8.0), $100 \mathrm{mM} \mathrm{NaCl}, 1 \%$ (w/v) Triton X-100 and centrifuged at $100000 \mathrm{~g}$ for $15 \mathrm{~min}$ at $4{ }^{\circ} \mathrm{C}$. The supernatant was incubated with $1.0 \mu \mathrm{l}$ of anti-Myc serum fixed on $7.5 \mu \mathrm{l}$ of protein G Sepharose 4 fast-flow beads. After the beads were washed, the precipitates were analyzed by SDSPAGE and immunoblotted with either anti-Myc or anti-FLAG antibody.

\section{GST Pulldown Assay}

COS-7 cells were transfected by DEAE-dextran with various Myc-tagged constructs of MAGI-1 or with FLAG-tagged nephrin. Cells from one $10 \mathrm{~cm}$ plate were homogenized in $200 \mu \mathrm{l}$ of $25 \mathrm{mM}$ Tris-HCl (pH 8.0), $100 \mathrm{mM} \mathrm{NaCl}, 1 \%(\mathrm{w} / \mathrm{v}$ ) Triton X-100, and were centrifuged at $100000 \mathrm{~g}$ for $15 \mathrm{~min}$ at $4^{\circ} \mathrm{C}$. The supernatant was used for assay. For each extract, $180 \mu \mathrm{l}$ was incubated with either $200 \mathrm{pmol}$ of various GST-nephrin proteins or GST-MAGI-1 proteins fixed on $10 \mu \mathrm{l}$ of glutathione beads. After the beads were washed, the proteins on the beads were immunoblotted with anti-Myc or anti-FLAG antibody.

\section{Induction of PAN Nephrosis}

All procedures related to the care and treatment of animals were in accordance with institutional and National Institutes of Health guideline. Young male rats $(150 \mathrm{~g})$ were injected intraperitoneally with $10 \mathrm{mg} / 100 \mathrm{~g}$ body weight of PAN (Sigma-Aldrich). Animals were killed on day 10 for immunoprecipitation from rat glomeruli, and on days 2, 5, 7, and 11 for immunofluorescence, and on day 11 for immunoelectron microscopic study.

\section{Immunoprecipitation from Rat Glomeruli}

Glomeruli were obtained from rat using sieving techniques. Renal cortex were minced and passed through $250 \mu \mathrm{m}-, \quad 150 \mu \mathrm{m}-$, and $75 \mu \mathrm{m}$-stainless meshes, sequentially. Glomeruli trapped by the last mesh were confirmed by microscopy and used as samples. MAGI-1 was immunoprecipitated from the $1 \%$ Triton X-100 and 1\% deoxycholic acid extract of isolated rat glomeruli. The immunoprecipitates were immunoblotted with either anti-MAGI-1 or anti-nephrin antibody.

\section{Immunohistochemistry}

Immunofluorescence studies were performed as described previously. ${ }^{36}$ Briefly, rat kidneys were perfused with $2 \%$ paraformaldehyde fixative buffered with $0.1 \mathrm{M}$ phosphate buffer (PB, $\mathrm{pH}$ 7.4). These samples were immersed in the same fixative for about $30 \mathrm{~min}$. After washing with phosphatebuffered saline (PBS), the tissue was immersed successively in PBS solution containing 10, 15, and $20 \%$ sucrose. After the tissue was embedded in OCT compound and frozen, cryosections (thickness 5-10 $\mu \mathrm{m}$ ) were cut using a Jung Frigocut 2800E (Leica) and then mounted on silane-coated glass slides. The cryosections were rinsed with PBS and blocked in blocking solution $(0.1 \%$ bovine serun albumin (BSA) in PBS). The sections were incubated with various first antibodies and visualized with rhodamine- or fluorescein isothiocyanate-conjugated second antibodies (Jackson ImmunoResearch Laboratories). Fluorescence specimens were viewed with a confocal laser scanning microscope LSM510 (Carl Zeiss).

\section{Immunoelectron Microscopy}

The samples fixed with paraformaldehyde as abovementioned were rinsed and infiltrated with $40 \%$ polyvinylpyrrolidone/2.3 $\mathrm{M}$ sucrose buffered with $0.1 \mathrm{M} \mathrm{PB}$ and embedded on nails for immunogold electron microscopy. Ultrathin cryosections were cut with a Leica Ultracut UCT microtome equipped with the FCS cryoattachment (Wein, Austria) at $-110^{\circ} \mathrm{C}$. Sections were transferred to nickel grids 
(150 mesh) that had been coated with a Formvar membrane. Subsequent incubation steps were carried out by floating the grids on droplets of the filtered solution. After quenching free aldehyde groups with PBS-0.01M glycine, sections were incubated overnight with various primary antibodies. They were then incubated with anti-mouse IgG coupled to 5 -nm colloidal gold (1:100) and/or antirabbit IgG coupled to $10-\mathrm{nm}$ colloidal gold (1:100) for $1 \mathrm{~h}$. Goat anti-rabbit IgG coupled to $10 \mathrm{~nm}$ colloidal gold and goat anti-mouse IgG coupled to $5 \mathrm{~nm}$ colloidal gold were obtained from British BioCell (Cardiff, UK). After immunostaining, they were fixed with $2.5 \%$ glutaraldehyde buffered with $0.1 \mathrm{M}$ PB. The sections were then contrasted with $2 \%$ uranyl acetate solution for $20 \mathrm{~min}$ and absorption-stained with $3 \%$ polyvinyl alcohol containing $0.2 \%$ uranyl acetate for $10 \mathrm{~min}$. As the negative control experiment, the primary antibodies were either omitted from the incubation solution or substituted by normal mouse IgG or normal rabbit IgG (Vector Laboratories) at the same concentrations as the primary antibodies.

\section{Results}

\section{Identification of Nephrin as a Novel Interacting Protein for MAGI-1}

In search for a novel interacting protein with MAGI1 , we performed yeast two-hybrid screening using human kidney cDNA library with PDZ1 to PDZ5 domains of MAGI-1 as a bait. We obtained 95 clones. Among them, pPrey 19237 encodes the C-terminal 496 amino acids of nephrin. To confirm the interaction between MAGI-1 and nephrin, we first attempted to co-immunoprecipitate MAGI-1 and nephrin from normal rat glomeruli. However, as we reported previously, MAGI-1 was highly resistant to the detergent extractions (Figure 1a). MAGI-1 could not be recovered even in the urea extraction (data not shown). Thereby, we could not test whether MAGI-1 and nephrin are co-immunoprecipitated from kidney lysates. Alternatively, we overexpressed Myc-MAGI-1 and FLAG-nephrin in COS-7 cells and immunoprecipitated Myc-MAGI-1 with anti-Myc antibody from the lysates. FLAGnephrin was co-precipitated with Myc-MAGI-1 (Figure 1b, lanes 1-4). The last four C-terminal amino-acid residues of nephrin are Gly-His-Leu-Val. This sequence does not fully match the consensus sequences of PDZ-binding motif. However, the last Val residue, which has been suggested to be the most important for the capacity to bind a PDZ domain, is conserved. ${ }^{37}$ It is therefore possible that nephrin interacts with PDZ domains of MAGI-1 by the Cterminus. To test this possibility, we overexpressed Myc-MAGI-1 and FLAG-nephrin-del C lacking the C-terminal five residues, in COS-7 cells and immunoprecipitated Myc-MAGI-1 with anti-Myc antibody from the lysates. FLAG-nephrin-del C

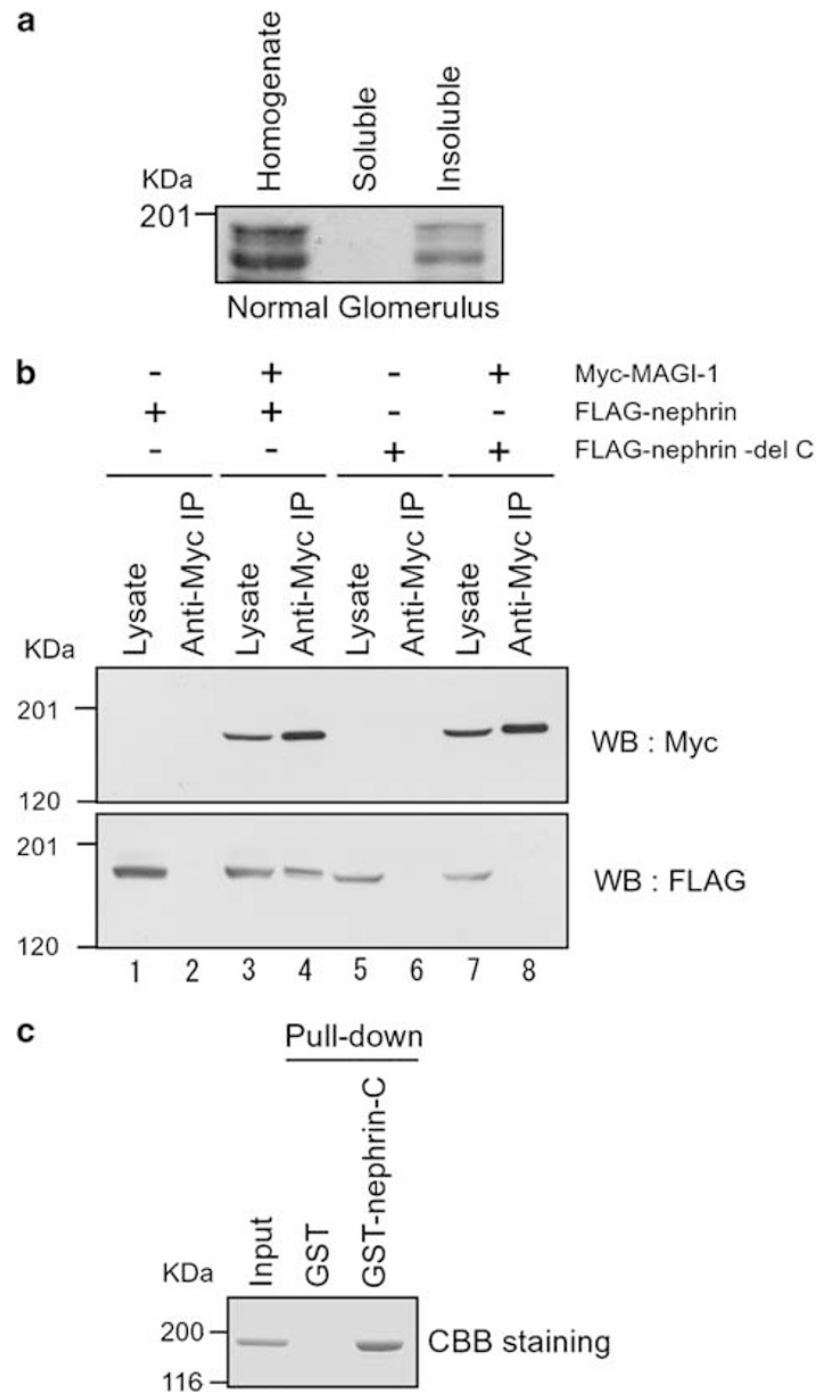

Figure 1 Interaction of nephrin with MAGI-1. (a) MAGI-1 from rat glomerular lysates is resistant to the detergent extraction. Rat glomerular preparations were lysed in buffers containing $1 \%$ Triton X-100 and 1\% deoxycholic acid. The resulting supernatant (Soluble) and pellet (Insoluble) fractions were immunoblotted with anti-MAGI-1 antibody. MAGI-1 was practically resistant to the extraction by $1 \%(\mathrm{w} / \mathrm{v})$ Triton X-100 and deoxycholic acid. (b) Co-immunoprecipitation of nephrin and MAGI-1. Myc-MAGI-1 with either FLAG-nephrin or FLAG-nephrin del C was coexpressed in COS-7 cells and immunoprecipitated by anti-Myc antibody. FLAG-nephrin but not FLAG-nephrin-del C was coprecipitated with Myc-MAGI-1. Protein standards are indicated on the left. Lanes 1, 3, 5, and 7; original inputs (Lysate). Lanes 2, 4, 6, and 8; immunoprecipitates with anti-Myc antibody (AntiMyc IP). (c) Direct interaction of MAGI-1 with nephrin in vitro. In all, 200 pmol of His-MAGI-1 was incubated with 250 pmol of either control GST or GST-nephrin-C immobilized on glutathioneSepharose beads. After the beads were washed, the precipitates were analyzed by SDS-PAGE and stained with Coomassie Brilliant Blue. His-MAGI-1 was efficiently trapped with GSTnephrin-C beads. $2 \mu \mathrm{g}$ of His-MAGI-1 was run as input control (Input). Protein standards are indicated on the left.

did not co-precipitate with Myc-MAGI-1, suggesting that the C-terminal sequence of nephrin is involved in the interaction (Figure 1b, lanes 5-8). 


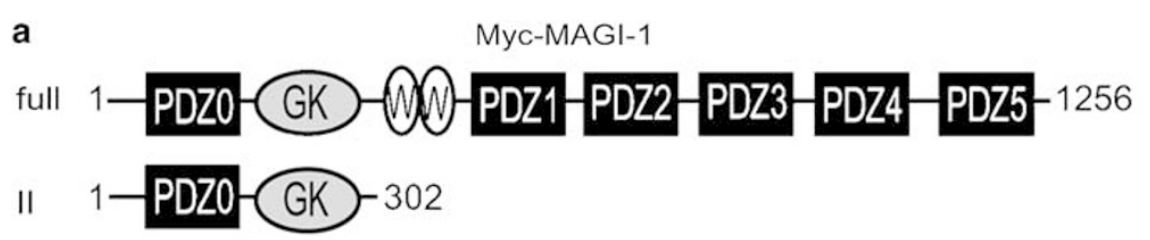

C

A 565-PDZ2-PDZ3-994

B

565-PDZ2-836

III

296- DUVI-PDZ1- 573

IV

565-PDZ2-PDZ3-994

V

975-PDZ4-PDZ5-1256

C

771-PDZ3-994

D $\quad 565-$ PDZ2 865

E

697-PDZ3-994

$\mathrm{F}$ b

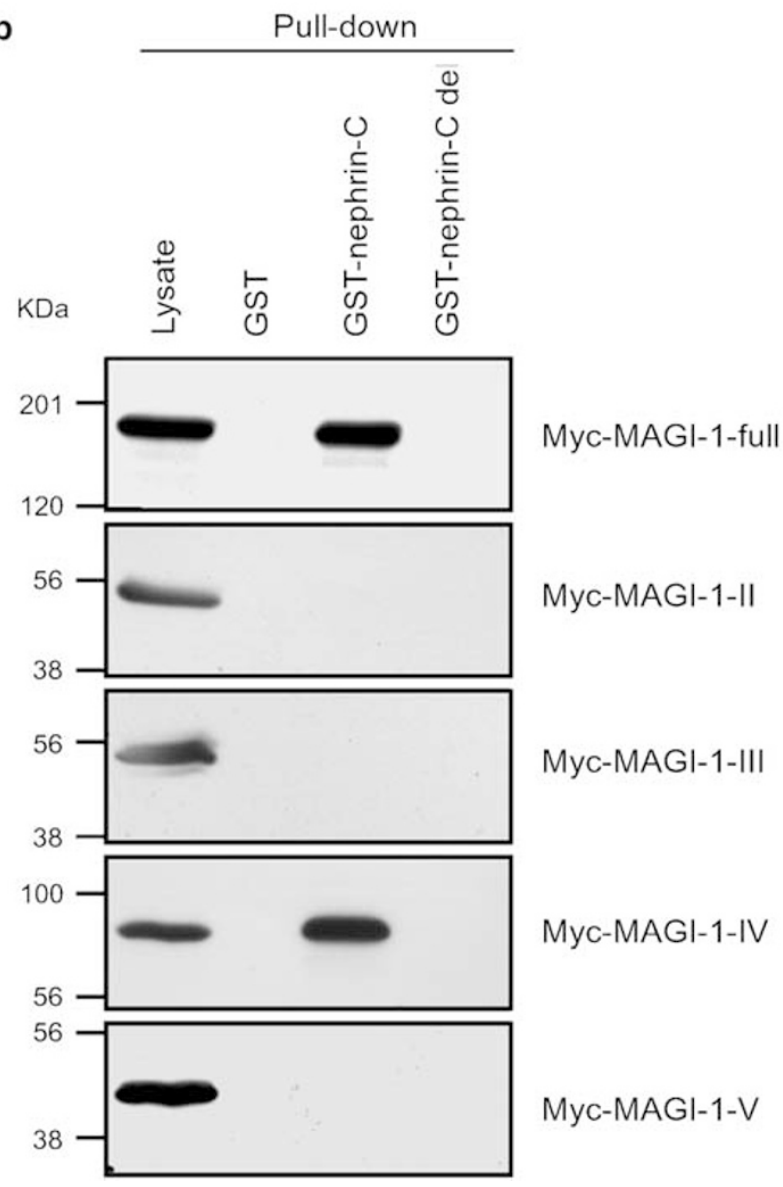

d

Pull-down
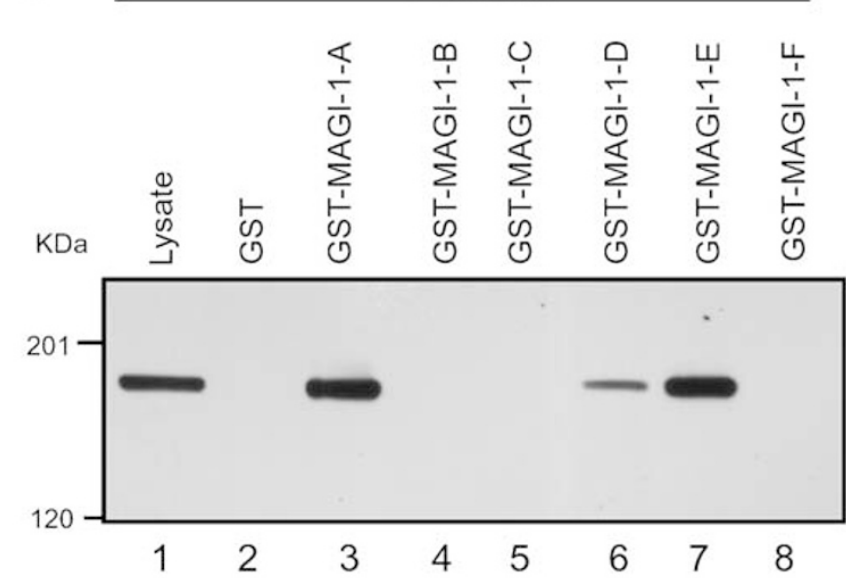

Figure 2 MAGI-1-interacting domain of nephrin. (a) Molecular organization of MAGI-1 and various Myc-MAGI-1 proteins. Numbers indicate the first and last amino-acid numbers of each Myc-MAGI-1. (b) Various Myc-tagged MAGI-1 proteins were expressed in COS-7 cells. The lysates $(20 \mathrm{mg}$ of total protein) were incubated with $250 \mathrm{pmol}$ of control GST or GST-nephrin-C immobilized on glutathioneSepharose beads. After the beads were washed, the precipitates were analyzed by SDS-PAGE and immunoblotted with anti-Myc antibody. GST-nephrin-C captured Myc-MAGI-1-full and Myc-MAGI-1-IV containing PDZ2 and PDZ3. Protein standards are indicated on the left. (c) Molecular organization of various GST-MAGI-1 proteins. Numbers indicate the first and last amino-acid numbers of each GSTMAGI-1. (d) FLAG-nephrin was expressed in COS-7 cells. The lysates were incubated with 250 pmol of various GST-MAGI-1 proteins immobilized on glutathione-Sepharose beads. After the beads were washed, the precipitates were analyzed by SDS-PAGE and immunoblotted with anti-FLAG antibody. GST-MAGI-1-A and -E efficiently trapped FLAG-nephrin. GST-MAGI-1-D only trapped a trace amount of FLAG-nephrin. GST, GST-MAGI-1-B, -C, or -F did not bind nephrin. These data suggest that nephrin binds to PDZ2 and PDZ3 of MAGI-1 but a single PDZ domain (PDZ2 or PDZ3) is not sufficient for efficient interaction. Protein standards are indicated on the left.

To examine whether MAGI-1 directly binds nephrin, we made use of His-MAGI-1 protein and GST-fusion protein containing the cytoplasmic region of nephrin (GST-nephrin-C). GST-nephrin-C trapped His-MAGI-1, whereas control GST did not (Figure 1c). 


\section{Nephrin-Interacting Domain of MAGI-1}

To identify the nephrin-interacting region of MAGI1 , we expressed various Myc-tagged MAGI-1 proteins in COS-7 cells and conducted pulldowns with GST-nephrin-C (Figure 2a). GST-nephrin-C captured Myc-MAGI-1-full and Myc-MAGI-1-IV containing PDZ2 and PDZ3 (Figure 2b). GST-nephrin-C lacking the C-terminal five residues (GST-nephrin-C del) did not bind to Myc-MAGI-1-full or Myc-MAGI-1-IV. Other Myc-MAGI-1 proteins were not precipitated by GST-nephrin-C. We next tried to determine which of PDZ2 and PDZ3 is involved in the interaction with nephrin. We expressed FLAGtagged nephrin in COS-7 cells and performed pulldowns using various GST-fusion proteins of MAGI-1 (Figure 2c). GST-fusion proteins containing either PDZ2 or PDZ3 (GST-MAGI-1-B or -C) did not bind nephrin (Figure 2d). However, GST-fusion protein containing PDZ2 with a part of PDZ3 (GST-MAGI-1D) trapped a trace amount of FLAG-nephrin. GSTMAGI-1-E containing PDZ3 with a part of PDZ2 more efficiently captured FLAG-nephrin. The region that is common to GST-MAGI-1-D and -E (GSTMAGI-1-F) did not bind nephrin. These results suggest that nephrin binds to PDZ2 and PDZ3 of MAGI-1 but a single PDZ domain (PDZ2 or PDZ3) is not sufficient for the efficient interaction.

\section{Nephrin does not Bind to ZO-1}

Neph1, a nephrin-like molecule with five extracellular Ig-like domains, has been reported to localize

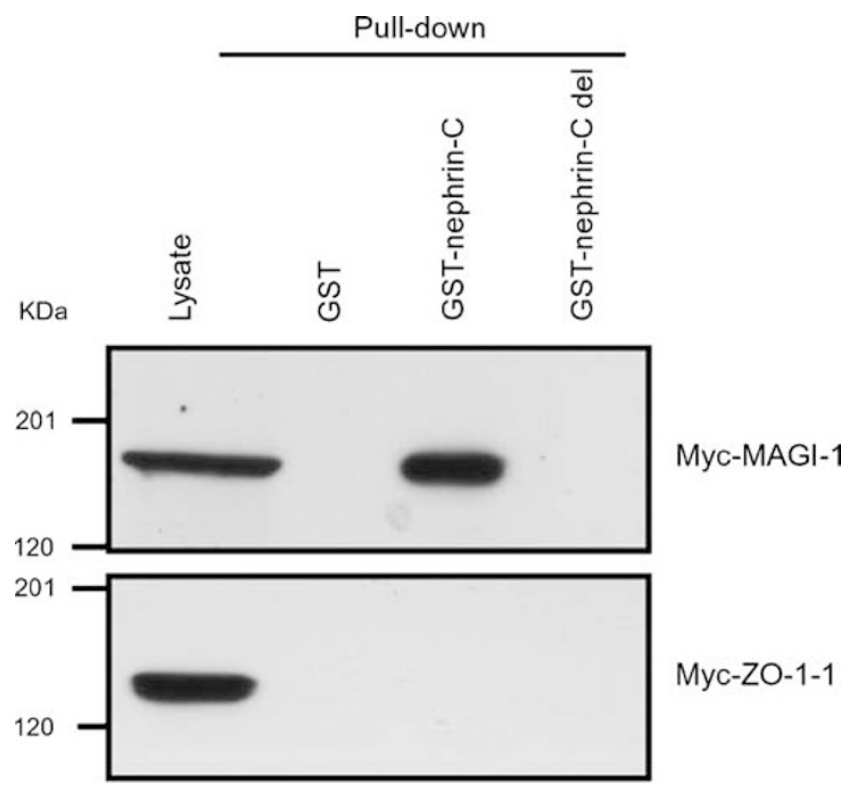

Figure 3 Specificity of the interaction of nephrin with MAGI-1. Lysates of COS-7 cells expressing Myc-MAGI-1 or Myc-ZO-1-1 were incubated with control GST or GST-nephrin-C immobilized on glutathione-Sepharose beads. The resulting complexes were analyzed by immunoblotting with anti-Myc antibody. Under the conditions where nephrin bound MAGI-1, nephrin did not bind ZO-1. Protein standards are indicated on the left. at the slit diaphragm. ${ }^{11-13}$ Neph1 binds to the PDZ domain of ZO-1 via its C-terminal PDZ-binding motif. $^{13,21}$ Next, we tested using GST pulldown assay whether nephrin also binds to not only MAGI-1 but also ZO-1. GST-nephrin-C did not bind Myc-ZO-1-1 under the condition in which it bound MAGI-1 in heterologous cells (Figure 3). Although this does not preclude the possibility that nephrin
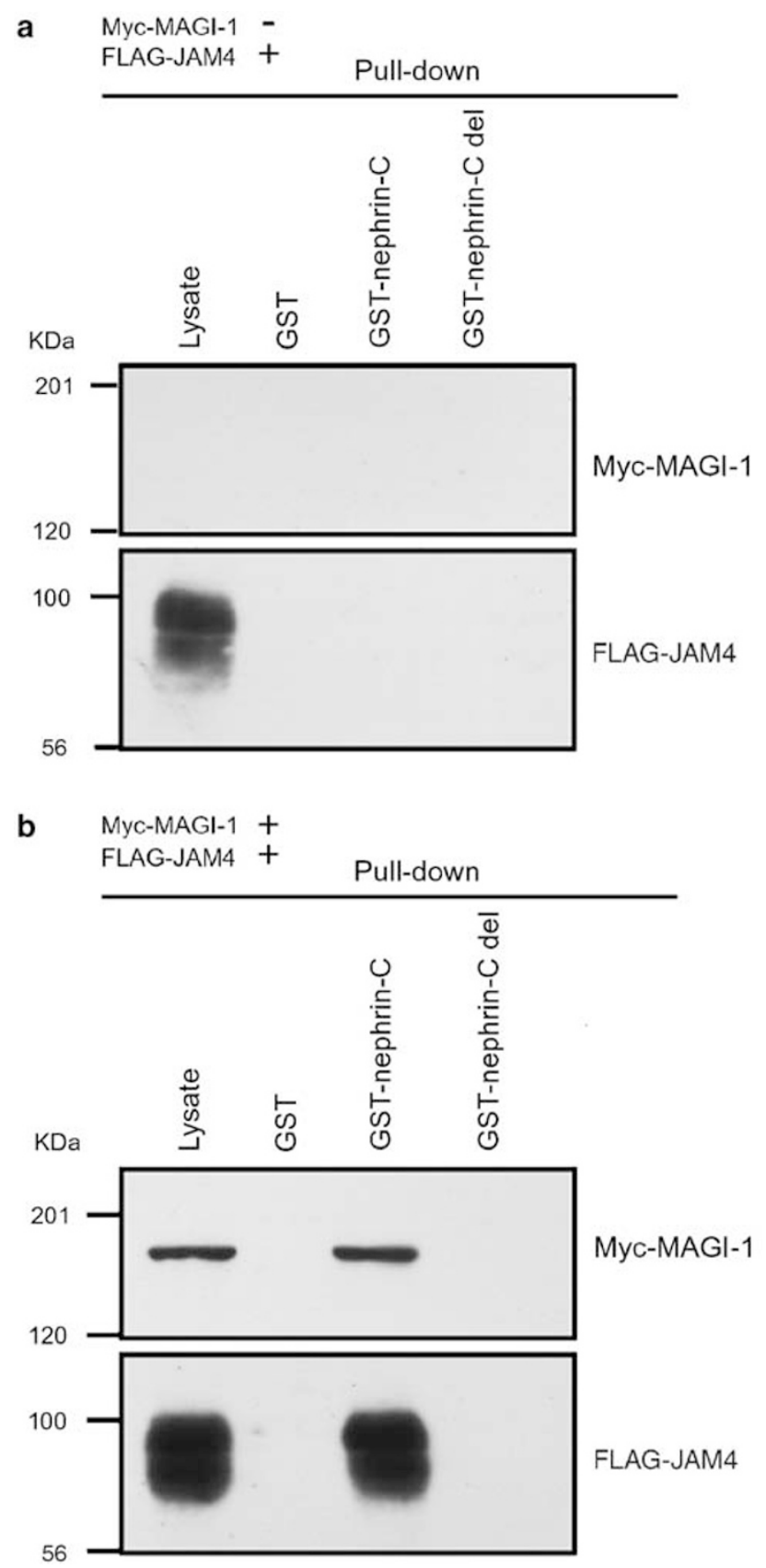

Figure 4 Tripartite complex of MAGI-1, nephrin, and JAM4. Lysates of COS-7 cells expressing FLAG-JAM4 alone (a) or together with Myc-MAGI-1 (b) were incubated with 250 pmol of either control GST or GST-nephrin-C immobilized on glutathioneSepharose beads. After the beads were washed, the precipitates were immunoblotted with anti-FLAG and anti-Myc antibodies. GST-nephrin-C trapped FLAG-JAM4 only in the presence of MycMAGI-1. Protein standards are indicated on the left. 
a

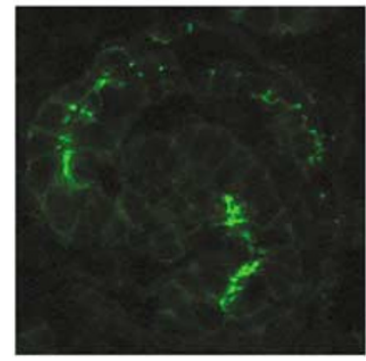

b

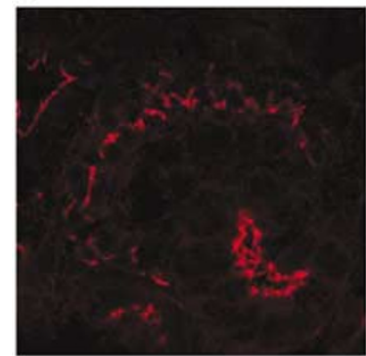

c

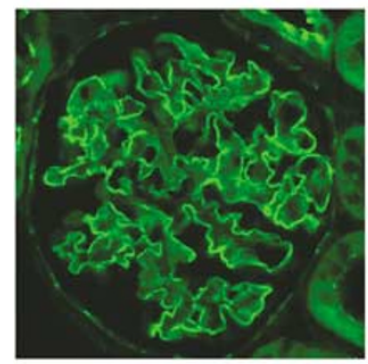

d

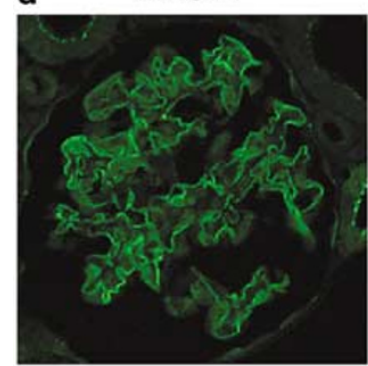

e

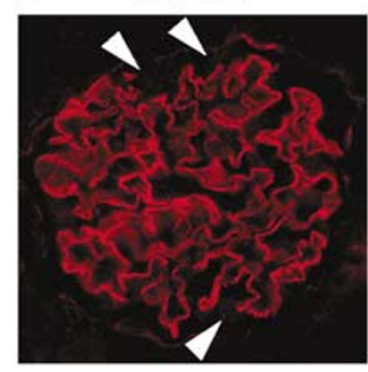

ZO-1

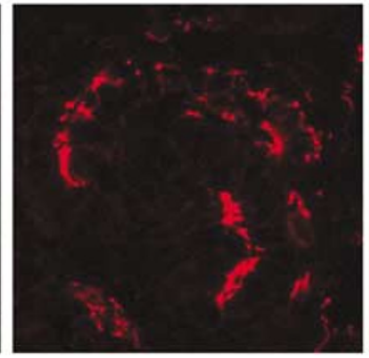

Podocalyxin

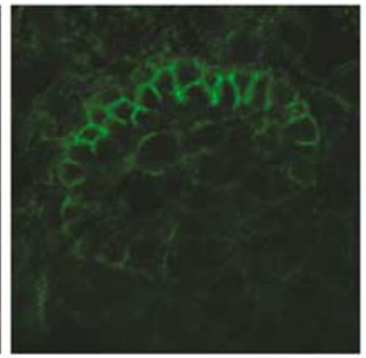

Nephrin

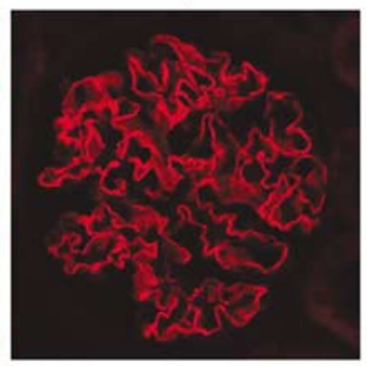

ZO-1

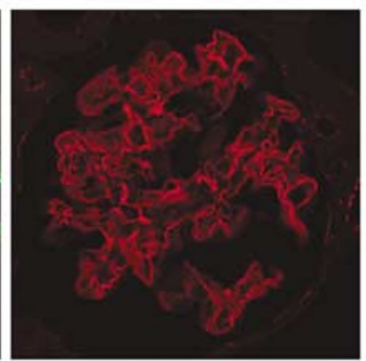

Podocalyxin

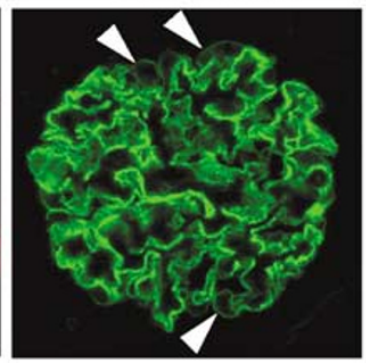

Merge

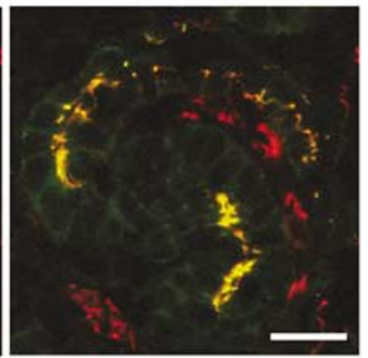

Merge

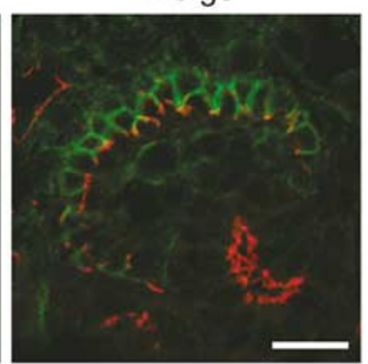

Merge

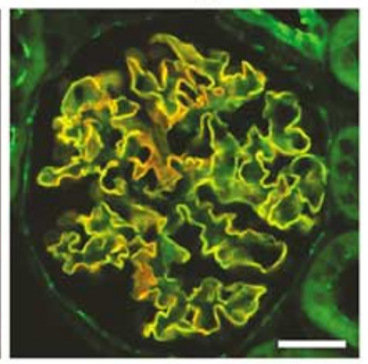

Merge

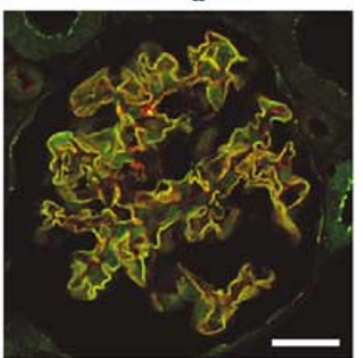

Merge

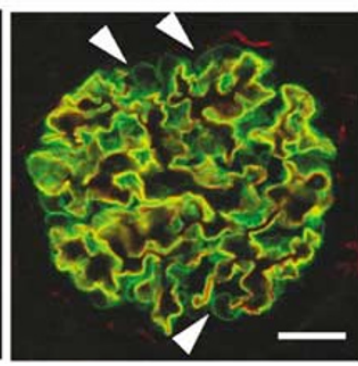

Figure 5 Immunohistological analysis of MAGI-1 in developing and mature podocytes. (a and b) MAGI-1 in developing podocytes. The sections of rat kidney were double stained with various antibodies. Bars, $20 \mu \mathrm{m}$. (a) MAGI-1 and ZO-1. (b) MAGI-1 and podocalyxin. MAGI-1 colocalized with ZO-1 at the site of tight junction. The localization of MAGI-1 is different from that of podocalyxin, which is located at the apical membrane. (c-e) MAGI-1 in mature podocytes. The sections of rat kidney were double stained with various antibodies. Bars, $50 \mu \mathrm{m}$. (c) MAGI-1 and nephrin. (d) MAGI-1 and ZO-1. (e) MAGI-1 and podocalyxin. MAGI-1 colocalized well with nephrin and ZO-1. MAGI-1 signal was not seen at the cell body of podocytes, where podocalyxin immunoreactivity was positive (arrowheads). 
indirectly interacts with ZO-1 to form a complex in the kidney, the above data imply that nephrin directly binds MAGI-1 but not ZO-1.

\section{MAGI-1, Nephrin and JAM4 Form a Tripartite Complex}

As we previously reported, JAM4 binds to PDZ1 and PDZ4 of MAGI-1. Since nephrin binds to the region containing PDZ2 and PDZ3 of MAGI-1, we speculated that MAGI-1 can bind to nephrin and JAM4 at the same time. We expressed FLAG-JAM4 alone or together with Myc-MAGI-1 in COS-7 cells and performed GST pulldown assays. GST-nephrin-C did not directly bind FLAG-JAM4 (Figure 4a). However, when Myc-MAGI-1 was coexpressed, FLAG-JAM4 was trapped with Myc-MAGI-1 by GST-nephrin-C (Figure 4b). This indicates that MAGI-1, nephrin, and JAM4 form a tripartite complex in vitro.

\section{Localization of MAGI-1, Nephrin, and JAM4 in Rat Glomeruli}

In the next set of experiments, we compared the localization of MAGI-1 with those of other proteins in podocytes. In the developing glomeruli, MAGI-1 colocalized with ZO-1, a component of the cytoplasmic face of the slit diaphragm at the junctional complexes (Figure 5a). The localization of MAGI-1 is different from that of podocalyxin, a marker of apical membrane of podocytes (Figure 5b). The signal for podocalyxin was stopped at the site of junctional complex containing MAGI-1. In the mature glomeruli, the immunofluorescence signal for MAGI-1 was detected as a linear staining pattern along the glomerular basement membrane and colocalized perfectly to that of nephrin and ZO-1, suggesting that MAGI-1 was located at the level of foot processes of podocytes (Figure $5 \mathrm{c}$ and $\mathrm{d}$ ). MAGI-1 immunostaining was not detectable at the cell body of podocytes, where podocalyxin immunoreactivity was positive (Figure 5e, arrowheads). The precise localization of MAGI-1 was detected by immunogold labeling of ultrathin frozen sections. We used double-label immunoelectron microscopy to detect nephrin and MAGI-1. In a previous paper, it was reported that a small portion of nephrin is localized in the cholesterol-rich apical region, based on biochemical fraction experiments. ${ }^{38}$ In developing podocytes, 5-1-6 mouse monoclonal antibody was localized at the basolateral membrane. ${ }^{39}$ However, in our immunoelectron microscopy, 5-1-6 mouse monoclonal antibody detected nephrin at the slit diaphragm in mature podocyte as reported previsously. ${ }^{33}$ MAGI-1 was likewise detected at the cytoplasmic surface of the slit diaphragm (Figure 6). We also compared the localization of JAM4 with that of ZO-1 and podocalyxin. The immunoreactivity of JAM4 colocalized primarily with ZO-1 in podocytes
(Figure 7a). However, JAM4 signals were also seen at the cell body of the podocytes, where ZO-1 immunoreactivity was negative (Figure 7a, arrowheads). JAM4 at the cell body of podocytes colocalized well with podocalyxin (Figure 7b). These immunofluorescence data suggest that in podocytes, MAGI-1 is localized exclusively at the foot processes, while the distribution of JAM4 extends to the apical surface. To confirm the localization of JAM4 in podocytes, we used immunogold electron microscopy with ultrathin frozen sections. JAM4 was localized both at the level of slit diaphragm and at the apical membrane of podocytes (Figure 7c).

\section{Localization of MAGI-1 in Nephritic Kidney}

Next, we assessed the localization of MAGI-1 at proteinuric states. PAN induces nephrosis with the
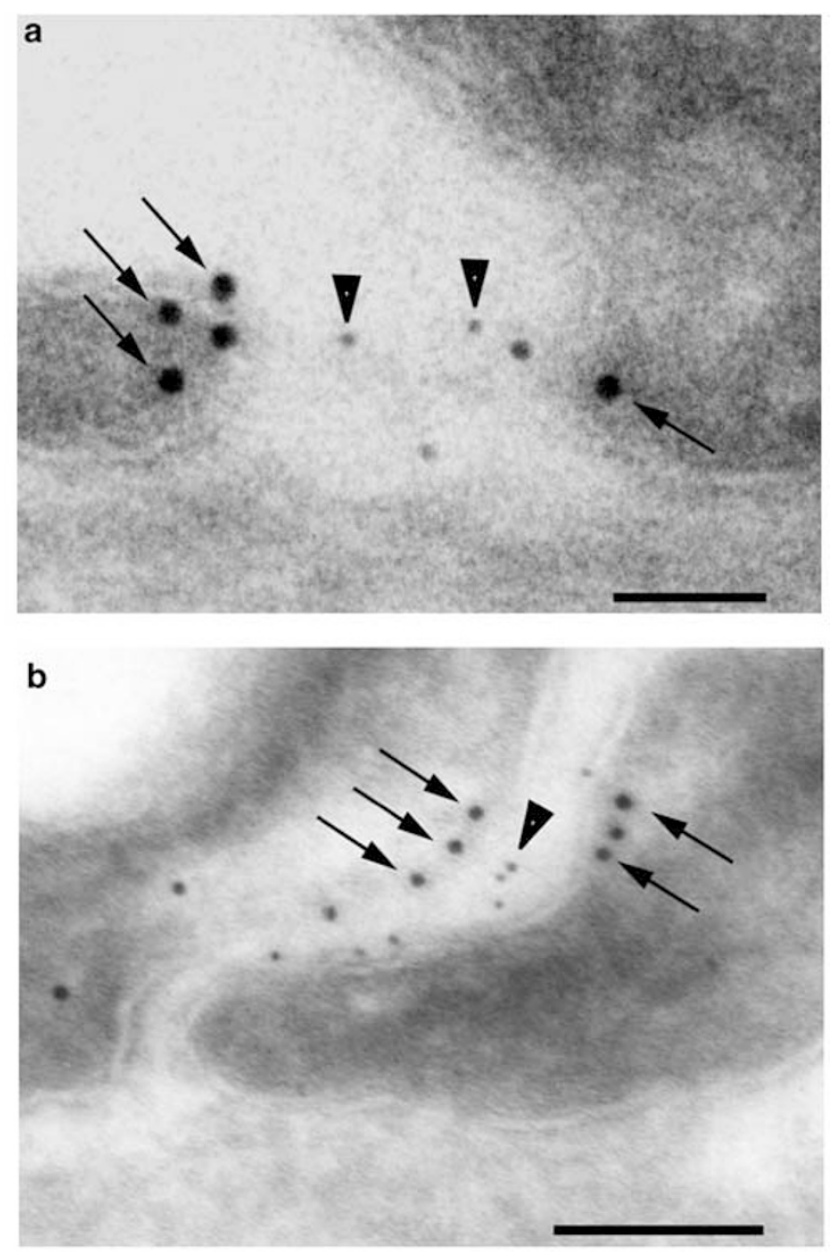

Figure 6 Immunogold localization of MAGI-1 in podocytes. Double-label immunoglold staining of the glomerulus using anti-nephrin antibody mAb 5-1-6 ( $5 \mathrm{~nm}$ gold: arrowheads) and anti-MAGI-1 antibody (10 nm gold: arrows). Bars, $50 \mathrm{~nm} \mathrm{(a),}$ $100 \mathrm{~nm}$ (b). (a) Cross-section of adult rat kidney. Nephrin localized at the slit diaphragm and MAGI-1 was detected at the cytoplasmic surface of the slit diaphragm. (b) Tangential section shows exclusive labeling of MAGI-1 along the cytoplasmic base of the slit diaphragm. 
a

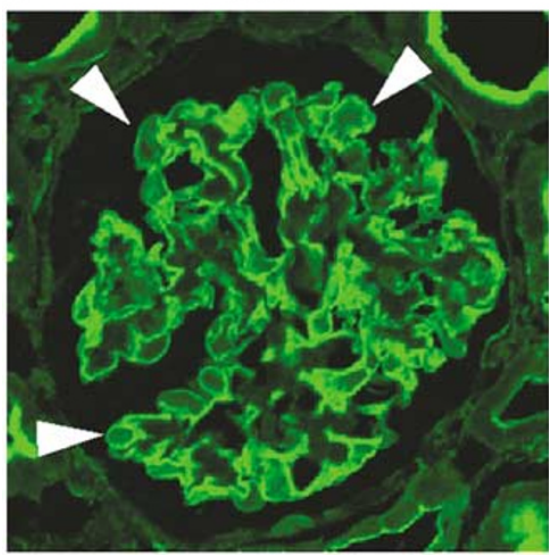

b

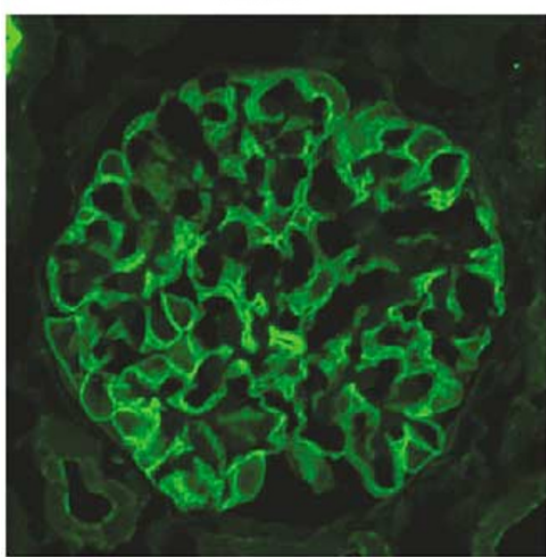

ZO-1

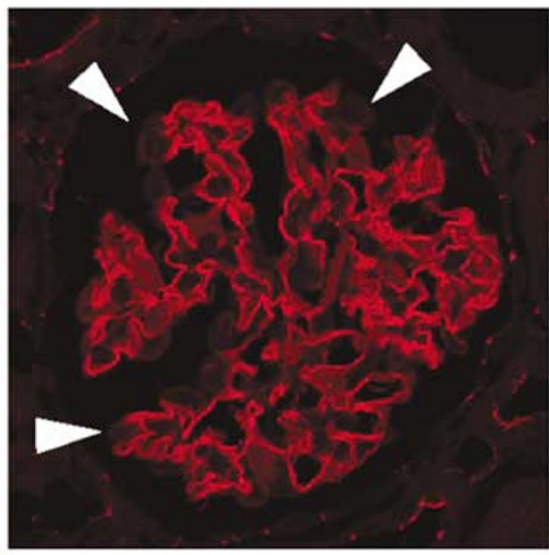

podocalyxin

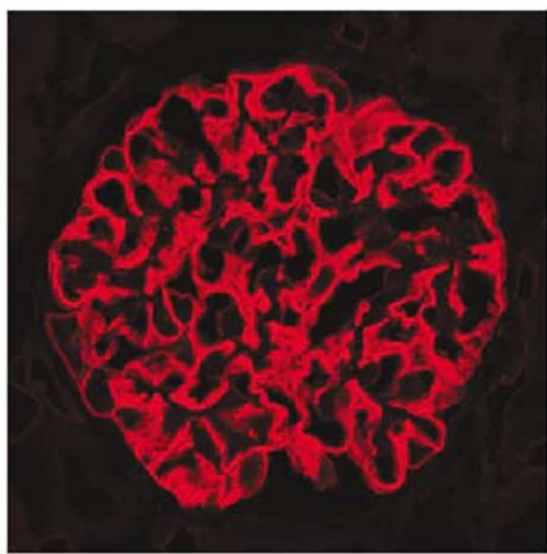

Merge

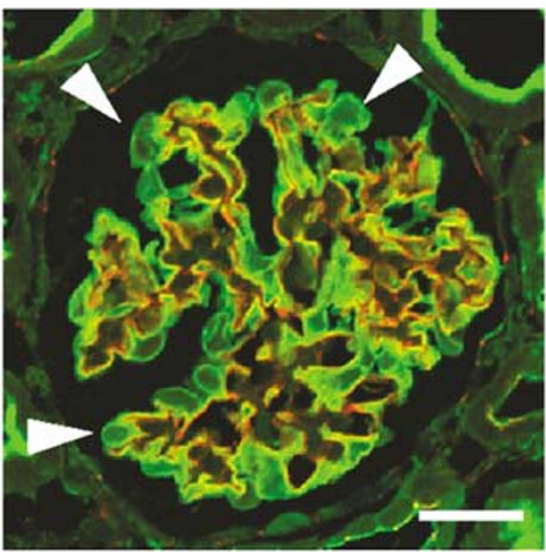

Merge

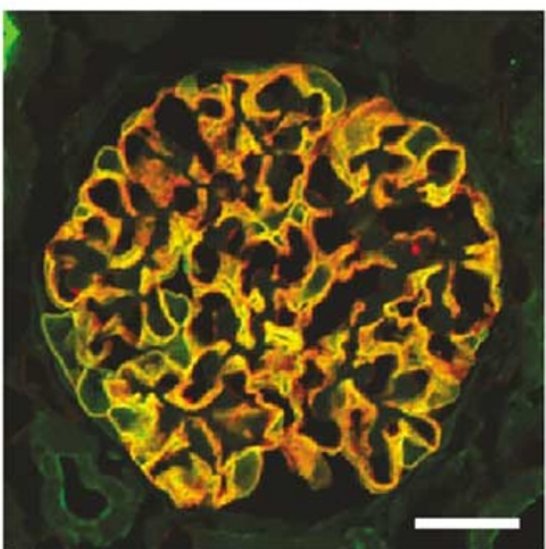

c

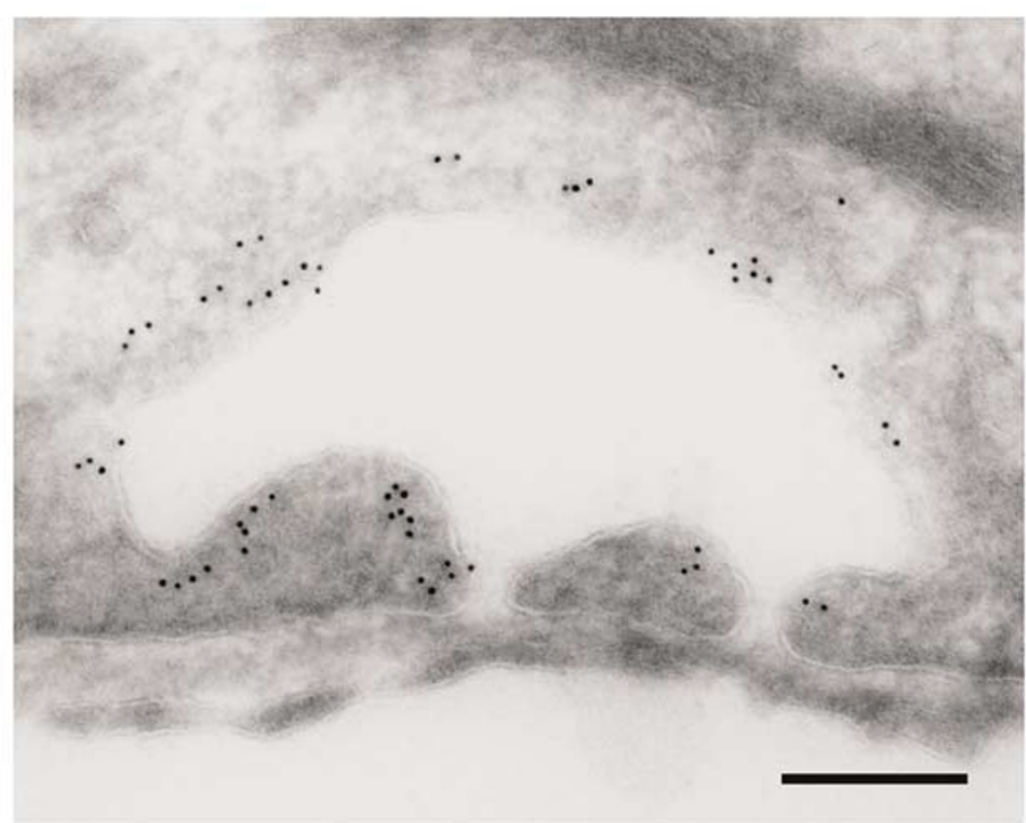

Figure 7 Immunohistological analysis of JAM4 in mature podocytes. (a and b) JAM4 in mature podocytes. The sections of rat kidney were double stained with various antibodies. Bars, $50 \mu \mathrm{m}$. (a) Immunofluorescence microscopy of JAM4 and ZO-1. The immunoreactivity of JAM4 was seen to colocalize with ZO-1. However, JAM4 was also detected at the cell body, where ZO-1 immunoreactivity was negative (arrowheads). (b) Immunofluorescence microscopy of JAM4 and podocalyxin. The signal for JAM4 is completely merged with that of podocalyxin, an apical marker protein of podocyte. (c) Immuoelectron microscopy of JAM4 in podocytes. JAM4 is localized not only at the slit diaphragm, but is widely distributed at the apical surface of the podocyte foot processes and the cell body. Bar, $0.2 \mu \mathrm{m}$. 
effacement of podocyte foot processes and changes nephrin and podocin distribution. ${ }^{40}$ Podocin is recruited to newly formed occluding-type junctions, while nephrin is not. ${ }^{29}$ Similarly, in passive Heymann nephritis (PHN), nephrin distribution becomes from a linear pattern to a clustered pattern. ${ }^{28}$ Nephrin dissociates from actin, and the slit diaphragm disappears or is displaced and replaced by occluding-type junctions. In contrast, CD2AP is retained in podocyte foot processes. To determine how MAGI-1 localization changes in nephrotic state, we induced PAN nephrosis in rats. On day 5 of the PAN injection, the staining pattern of MAGI-1 changed to a coarse granular pattern, which correlated with the staining pattern of nephrin (Figure 8a). On day 7, the staining intensity of MAGI-1 and nephrin was decreased (Figure 8a). On day 11, the staining intensity of both MAGI-1 and nephrin was dramatically decreased, and almost no detectable staining was seen in the glomerulus (Figure 8a). In contrast, no changes in the staining pattern and intensity were detected in the staining of ZO- 1 (Figure $8 \mathrm{~b}$ ). The immunogold electron microscopy with ultrathin frozen sections revealed the effacement of foot processes and newly formed occluding-type junctions. Nephrin immunoreactivity was detected at the dislocated slit diaphragm above the newly formed junction (Figure 8d). MAGI-1 immunoreactivity retained at the cytoplasmic surface of the dislocated slit diaphragm and was not detected at occluding-type junctions (Figure 8c and $d$ ). These data suggest that MAGI-1, unlike CD2AP and podocin, is tightly associated with slit diaphragm even at the proteinuric states. In addition, gold particles for JAM4 were distributed above the dislocated slit diaphragm (Figure 8e).

\section{MAGI-1 Binds Nephrin In Vivo}

Finally, we tested whether we could immunoprecipitate MAGI-1 and nephrin from the glomeruli of PAN-treated rat. In contrast to the normal state of glomeruli, a fraction of MAGI-1 became soluble in the detergents including Triton X-100 and deoxycholic acid on day 10 of PAN treatment (Figures 1a and $8 \mathrm{f}$ ). We immunoprecipitated MAGI-1 with antiMAGI-1 antibody. The antibody recognized three bands in the lysates and all these bands were immunoprecipitated. Although the identity of each band is not clear, we consider that these bands correspond to alternative splicing variants of MAGI-1. The immunoprecipitates with anti-MAGI-1 antibody contained nephrin (Figure 8g). This biochemical result also supports that the interaction between MAGI-1 and nephrin is maintained under the nephrotic state.

\section{Discussion}

The slit diaphragm between podocyte foot processes has a highly ordered structure and is proposed to form a zipper-like porous structure.$^{41}$ Recent studies have improved the knowledge of the molecular composition of the slit diaphragm complex. Three members of the Ig superfamily (nephrin, Neph1, and JAM4) and two members of the cadherin superfamily (P-cadherin and FAT) are associated with the slit diaphragm. .12,13,25,42,43 $^{1}$ All of them are cell adhesion molecules and interact with anchoring proteins at the cytoplasmic surface. Neph1 and JAM4 interact with ZO-1 and MAGI-1, respectively. ${ }^{13,21,25}$ Nephrin binds to CD2AP and podocin. ${ }^{5,7,15} \mathrm{~A}$ recent paper also reports the interaction between nephrin and CASK, a member of MAGUKs. ${ }^{44}$ Furthermore, the authors describe that Pcadherin forms a multiprotein complex containing nephrin, p120 catenin, CD2AP, and ZO-1, although it is unclear whether P-cadherin directly binds with these proteins at the slit diaphragm region. The intracellular binding partners for FAT in podocytes have not been identified yet. These anchoring proteins serve as adaptors to link transmembrane proteins to the actin cytoskeleton. Moreover, ZO-1, podocin, and CD2AP are proposed to be involved in the signalings of cell adhesion molecules. ${ }^{21,45,46}$ The significance of the interactions among slit diaphragm proteins and slit diaphragm-associated proteins is underscored by the fact that some molecular interactions are lost and the components redistribute in podocyte foot processes under the proteinuric state.

In this study, we have demonstrated a novel interaction between nephrin and MAGI-1. Biochemical

Figure 8 MAGI-1 in proteinuric state of podocytes. (a and $\mathbf{b}$ ) MAGI-1 in proteinuric podocytes induced by PAN injection. After indicated time periods of PAN injection, the sections of rat kidney were double stained with various antibodies. (a) MAGI-1 and nephrin. (b) MAGI1 and ZO-1. In the nephrotic states, the staining pattern of MAGI-1 changed to a coarse granular pattern, which correlated with the staining pattern of nephrin but not with that of ZO-1. Bar, $40 \mu \mathrm{m}$. (c) and (d) Immunoelectron microscopy of MAGI-1 labeled with $10 \mathrm{~nm}$ gold particles in PAN nephrotic podocyte. MAGI-1 $(10 \mathrm{~nm}$ colloidal gold) is located at the base of the dislocated slit diaphragm (arrowhead) labeled with anti-rat nephrin antibody (5-1-6, $5 \mathrm{~nm}$ colloidal gold) above the newly formed junction (arrow) between neighboring foot processes. Bar, $50 \mathrm{~nm}$. (e) Immunoelectron microscopy of JAM4 labeled with $10 \mathrm{~nm}$ gold particles in nephritic podocyte. JAM4 is distributed along the apical membrane above the dislocated slit diaphragm (arrowhead). Bar, 50 nm. (f) MAGI-1 from PANtreated rat glomeruli is soluble in the detergent extraction. PAN-treated rat glomerular preparations were lysed in buffers containing $1 \%$ Triton X-100 and 1\% deoxycholic acid. The resulting supernatant (Soluble) and pellet (Insoluble) fractions were immunoblotted with anti-MAGI-1 antibody. (g) Co-immunoprecipitation of MAGI-1 and nephrin from rat glomerulus of the proteinuric state. Glomeruli were isolated from rats treated with PAN. Lysates were incubated with either preimmune or anti-MAGI-1 antibody immobilized on protein G Sepharose beads. After the beads were washed, the precipitates were analyzed by SDS-PAGE and immunoblotted with anti-MAGI-1 and anti-nephrin antibodies. The lysate used for immunoprecipitation was run as input control (Lysate). Protein standards are indicated on the left. 
a

MAGI-1

Nephrin

Merge
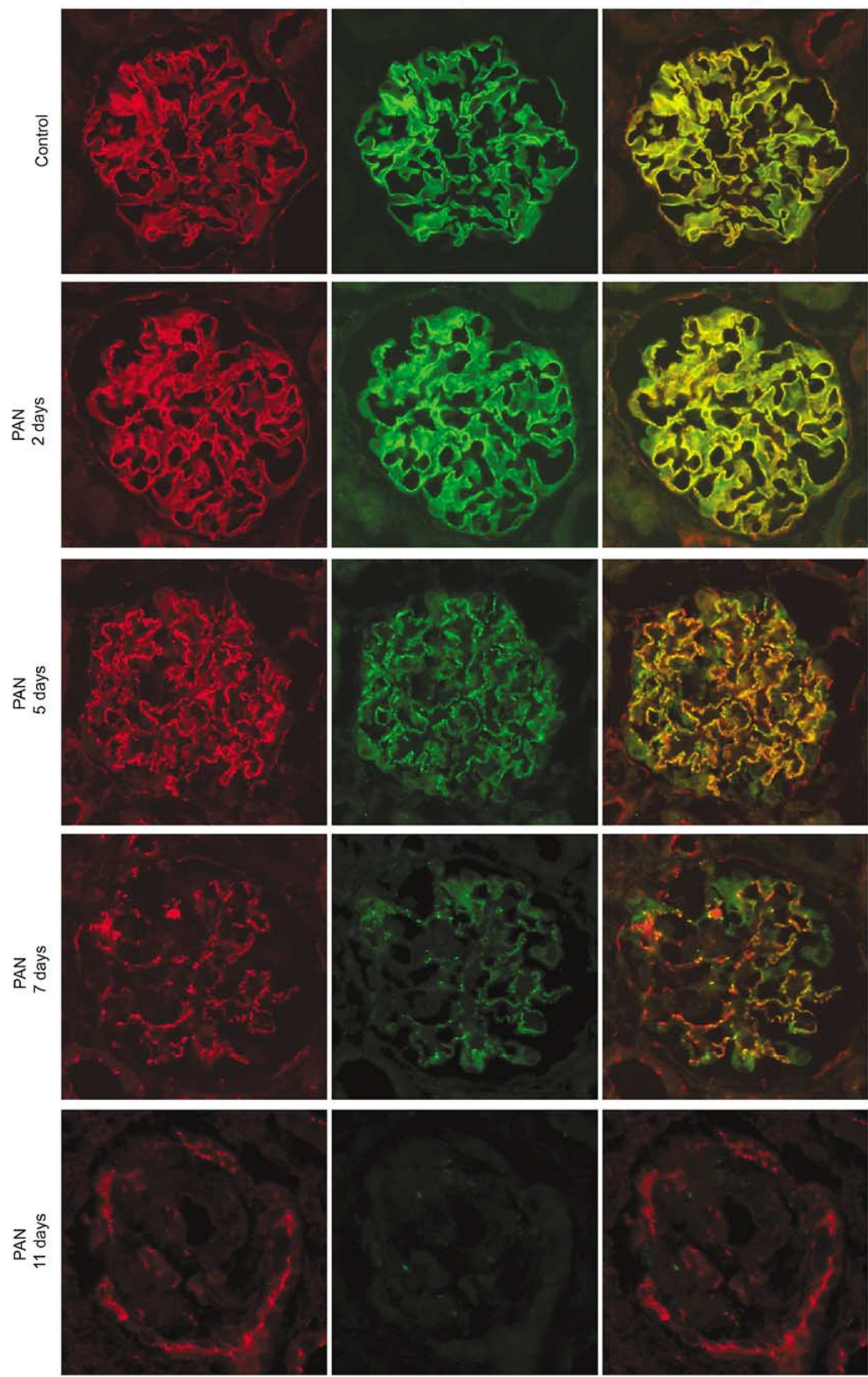
b
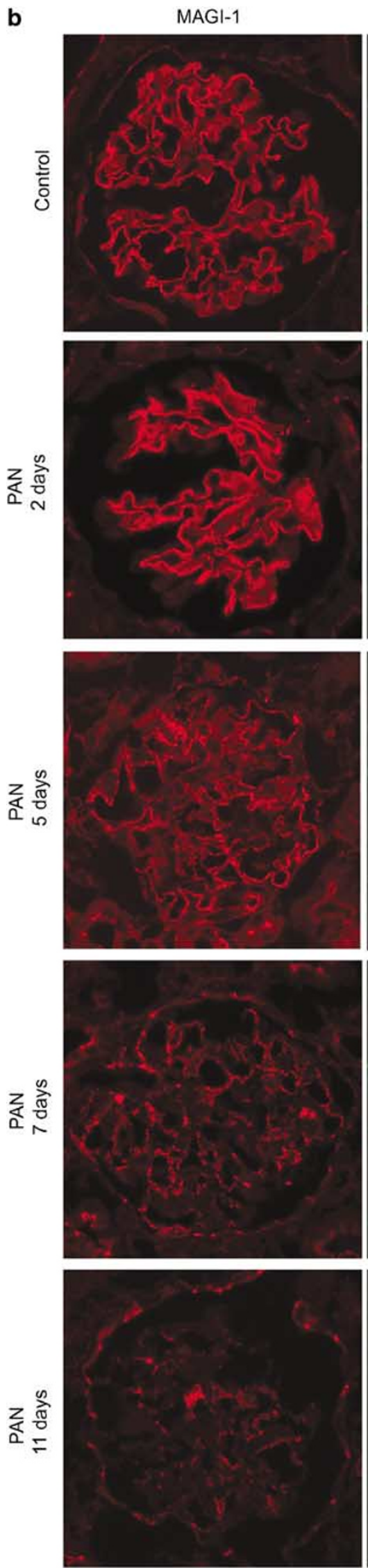

ZO-1
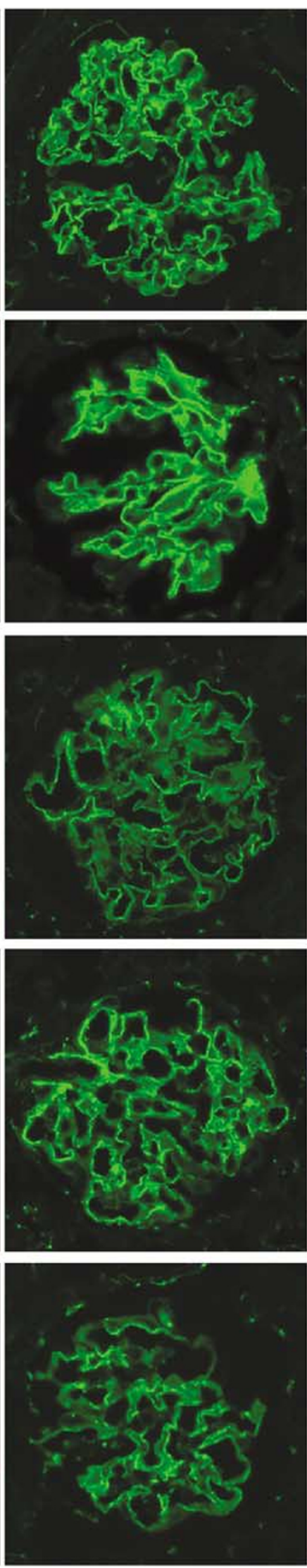

Merge
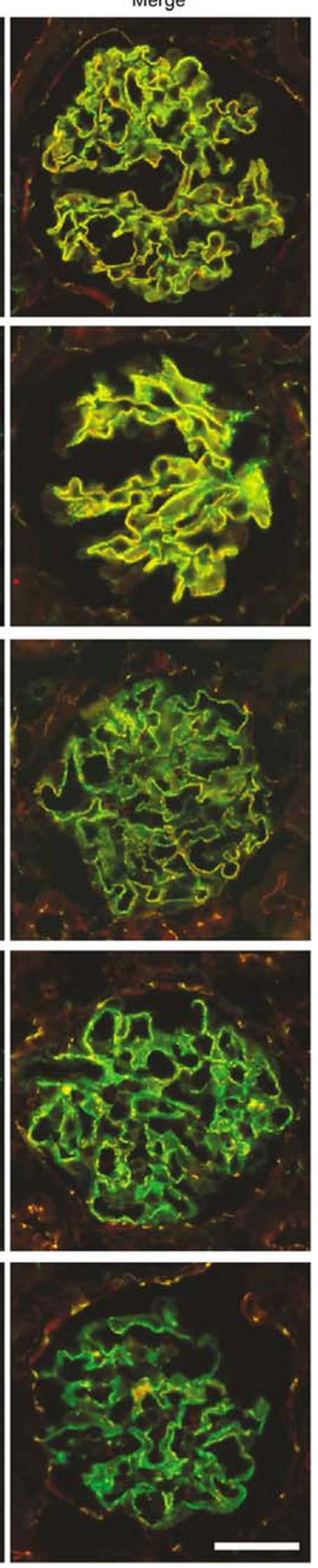

Figure 8 Continued. 

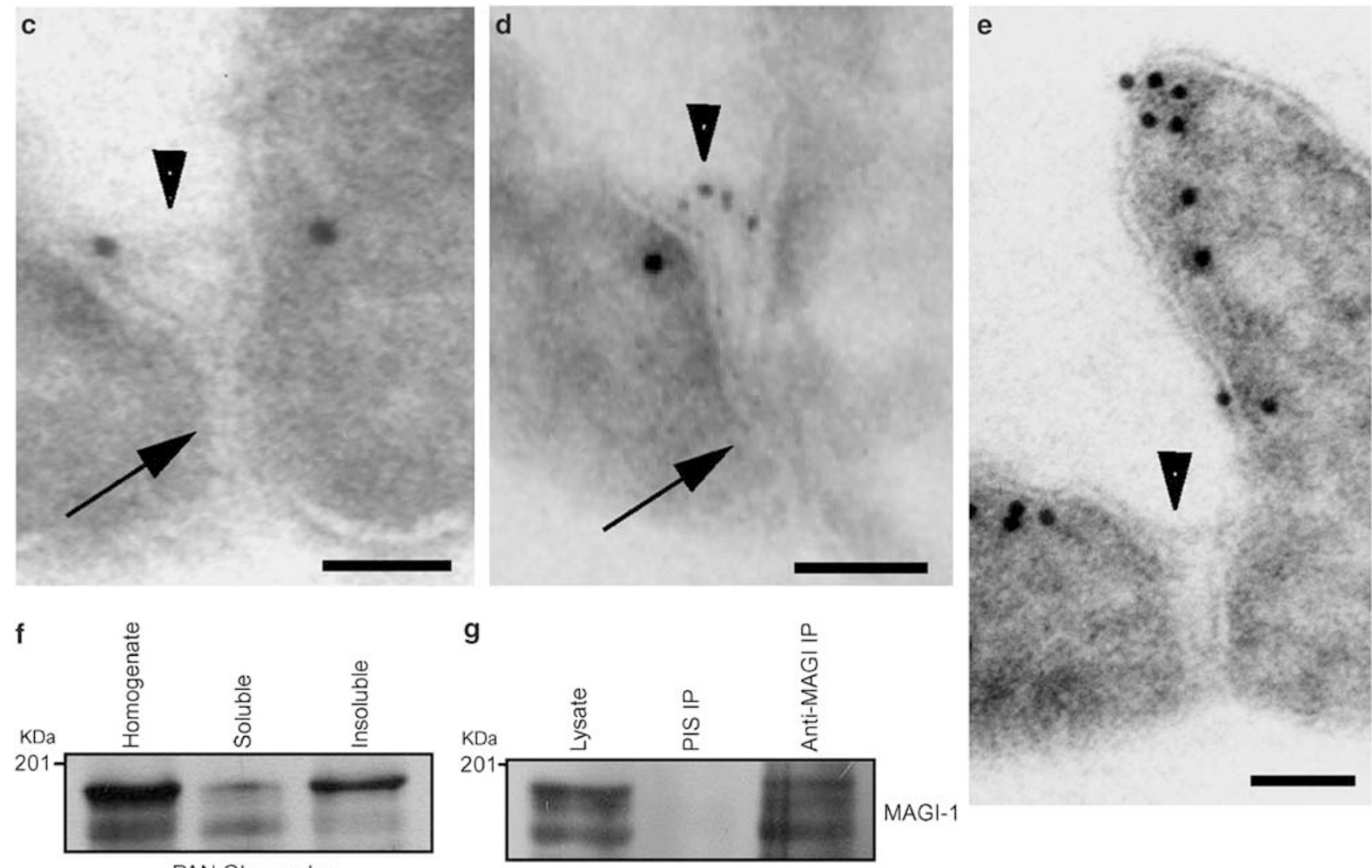

PAN Glomerulus

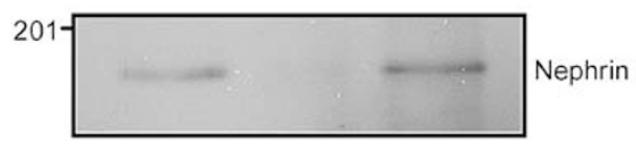

Figure 8 Continued.

experiments reveal that nephrin directly interacts with MAGI-1 via its C-terminus. The C-terminal four amino-acid residues of nephrin are Gly-His-Leu-Val, not consistent with type I, type II, or type III PDZbinding motifs. ${ }^{47}$ However, because the pKa of His is $\mathrm{pH}$ 6.5, it can be uncharged depending on the local environment and the sequence may behave as a type II motif. This idea is consistent with the recent report that nephrin binds to CASK, because type II motif is a ligand for the PDZ domain of CASK. Nephrin binds to the region covering tandem PDZ2 and PDZ3 domains of MAGI-1. PDZ2 or PDZ3 alone is not sufficient for the interaction. A novel mode of PDZ domain-mediated ligand binding is proposed for tandem PDZ domains of glutamate receptorinteracting protein (GRIP). ${ }^{48}$ Glutamate receptor subunits 2 and 3 require both PDZ4 and PDZ5 domains of GRIP for the efficient binding. As a matter of fact, PDZ5 is responsible for the binding. However, PDZ4 of GRIP needs to stabilize the structure of PDZ5. In the case of nephrin and MAGI-1, the data suggest that both of PDZ2 and PDZ3 can bind nephrin, although PDZ3 may be more efficient, but that the additional sequence besides PDZ domain itself is necessary for each PDZ domain to bind nephrin. It remains to be clarified whether two nephrin molecules simultaneously bind to MAGI-1.

In contrast to nephrin, JAM4 has canonical type I PDZ-binding motif (Val-Thr-Leu-Val). Consistently, JAM4 binds to MAGI-1 by different PDZ domains from nephrin, indicating that both nephrin and JAM4 can bind to MAGI-1 simultaneously in vitro. Among five cell adhesion molecules localized at the slit diaphragm, Neph1 and FAT also have type I motif (Gln-Thr-His-Val for Neph1 and His-Thr-GluVal for FAT). Recent studies show that Neph1 binds ZO-1 and we found that JAM4 does not bind to ZO1 , suggesting that the motifs of Neph1 and JAM4 may show different specificities, although both belong to type I. ${ }^{13,21,25}$ Therefore, the cell adhesion molecules at the slit diaphragm may interact with multi-PDZ proteins through distinct PDZ domains, and be assembled into the slit diaphragm. To understand the molecular structures of the slit diaphragm, it is important to make a complete list of multi-PDZ proteins associated with the slit diaphragm and determine which transmembrane protein is a ligand for each PDZ domain.

Although a previous study reports the presence of MAGI-1 in kidney glomeruli, its precise localization has not been determined. In the present study, we 
have shown that MAGI-1 is localized at the slit diaphragm. Thus, MAGI-1, like ZO-1, is a common component of the tight junction and the slit diaphragm. During revision of this manuscript, Lehtonen et $a l^{49}$ identified S-SCAM/MAGI-2 as a component of the nephrin multiprotein complex using mass spectrometry; however, the peptide sequences obtained in their analysis were not described. Since the rat MAGI-1 sequence is not currently in any sequence databases, it is difficult to conclude that they identified the peptide sequences specific for rat S-SCAM/MAGI-2 and that S-SCAM/ MAGI-2 is indeed expressed in the kidney. The antiMAGI-1 antibody used in our study was originally raised against S-SCAM/MAGI-2. Thereby, if SSCAM/MAGI-2 is expressed in podocytes, it is possible we would detect S-SCAM/MAGI-2 by immunohistochemistry and immunoelectron microscopy. However, S-SCAM/MAGI-2 message is detected only in the brain by Northern blots, while MAGI-1 transcript is detected ubiquitously in tissues including the kidney. ${ }^{22,50}$ Therefore, we postulate that the signal detected by our antibody in rat kidney reflects mainly MAGI-1 and not S-SCAM/MAGI-2.

One of the important functions of tight junction is to define the boundary between apical and basolateral plasma membrane domains. ${ }^{51}$ Recent studies have revealed that multiple protein complexes at tight junctions are important to determine the cell polarity. ${ }^{52}$ Although slit diaphragm does not act as a seal of the intercellular space like tight junction, it must be emphasized that slit diaphragm also serves as a fence between apical and basolateral membrane domain. As discussed above, the slit diaphragm is composed of cadherins and Ig superfamily proteins, but to our knowledge it is unclear how these proteins are involved in such a function. Podocalyxin, which is the major sialoglycoprotein of podocytes, is distributed along the apical membrane facing the urinary space and does not extend beyond the slit diaphragm. ${ }^{53,54}$ The current study clearly demonstrates that the localization of JAM4 in podocytes is overlapped by that of podocalyxin, a marker for apical membrane. This distribution is compatible with the localization of JAM4 in polarized epithelial cells. In confluent filter-grown Madin Darby canine kidney cells, JAM4 is surface-biotinylated only from the apical side, although JAM4 is detected at tight junctions. ${ }^{27}$ Moreover, overexpressed JAM4 is overflown to the apical membranes and JAM4 immunoreactivity is detected on apical membranes of proximal renal tubular and intestinal epithelial cells. All these findings imply that JAM4 is an apical membrane protein and is recruited to tight junctions from the apical side. We speculate that JAM4 is similarly recruited to the slit diaphragm from the apical side in podocytes. On the other hand, nephrin is a mainly localized and interacts with MAGI-1 at the level of slit diaphragm. ${ }^{55}$ PAN treatment triggers the displacement of the slit diaphragm and highlights its roles to separate the apical and basolateral membranes. In PAN nephritic kidney, podocalyxin was found exclusively apical to the displaced slit membrane. ${ }^{54}$ JAM4 is also found apical to the dislocated slit diaphragm containing nephrin. Therefore, the dislocated slit diaphragm still serves as the apical border. Most strikingly, MAGI-1 remains to be colocalized with nephrin at the dislocated slit diaphragm in PAN nephritic kidney, although the signals for both nephrin and MAGI-1 in glomeruli are remarkably decreased after the onset of proteinuria. Two nephrin-binding proteins, CD2AP and podocin, are dissociated from nephrin under the nephrotic state. CD2AP is retained in foot processes in a rat model of membrane nephropathy, while nephrin is diminished. ${ }^{28}$ Podocin is detected at the PAN-induced newly formed junction and is separated from nephrin that is attached to the delocalized slit diaphragm. ${ }^{29}$ Furthermore, ZO-1 has been shown to localize at both the slit diaphragm and newly formed junctional complexes. ${ }^{54}$ This indicates that MAGI-1 is unique among the slit diaphragm-associated proteins in that it sticks to the slit diaphragm even under the nephrotic state. These data suggest that nephrin and JAM4 assemble with MAGI-1 and those make a tripartite complex like in vitro at the slit diaphragm under both normal and pathological conditions. MAGI-1 may play a role in defining the edge of the apical plate by scaffolding the apical protein JAM4 and slit diaphragm protein nephrin at the same site.

The physiological significance of the interaction between MAGI-1 and nephrin remains to be elucidated. Scaffolding proteins such as CD2AP, ZO-1, and CASK are implicated in connecting nephrin to the cytoskeleton. ${ }^{38,44}$ Since MAGI-1 interacts with actin-binding proteins such as $\alpha$-actinin- 4 and synaptopodin, the role of MAGI-1 may similarly link nephrin to the cytoskeleton, as shown for JAM4. ${ }^{25}$ Membrane-bound MAGI-1 is extremely detergent-resistant. In PAN-treated glomeruli, however, MAGI-1 comes to be detergent-soluble, suggesting that MAGI-1 is no more linked to the cytoskeleton. Interestingly, MAGI-1 still interacts with nephrin in Triton X-100 and deoxycholic acidsoluble fraction, indicating that MAGI-1-nephrin complex dissociated from actin filaments is dispersed in the cytoplasm. The immunofluorescence signals for MAGI-1 and nephrin in the glomeruli were remarkably decreased 7 days after PAN injection, when the severe proteinuria was detectable. Yuan et $a l^{28}$ also reported that a progressive loss of nephrin staining is concerned with the dissociation of this molecule from actin cytoskeleton under the nephrotic condition. The loss of the molecular machinery to attach nephrin to the cytoskeleton may disrupt the filtration barrier. Recent studies shed light on the nephrin-involved signalings. Nephrin is phosphorylated by an Src family kinase, Fyn, and stimulates the AP-1 transcription factor 
through activation of mitogen-activated protein kinases. ${ }^{45,56}$ Nephrin also associates with p85 regulatory subunit of PI3K and stimulates PI3K-dependent AKT signaling in podocytes. ${ }^{46}$ In polarized epithelial cells, MAGI-1 interacts with several signaling molecules including RapGEP, $\beta$-catenin and mNET1. ${ }^{57-59}$ MAGI-1 may modulate nephrinmediated signaling in podocytes, although the roles of these signaling molecules at the podocytes are not known.

In conclusion, MAGI-1 is a slit diaphragmassociated protein that closely interacts with nephrin. MAGI-1 is well positioned to play roles as a scaffold protein at the slit diaphragm and connect nephrin and JAM4 to the actin cytoskeleton. As the mutations of nephrin and nephrin-interacting proteins including CD2AP and podocin cause nephrotic diseases, it will be important to determine whether MAGI-1 is similarly implicated in the pathogenesis of human kidney diseases.

\section{Acknowledgements}

We thank Dr Marilyn, G Farquhar (University of California, San Diego, CA, USA) for the antibody against podocalyxin and Dr Y Nakamura (University of Tokyo) for cDNA of human MAGI-1. This study was supported by grants-in-aids for Scientific Research and on Priority Areas, and Special Coordination Funds for Promoting Science and Technology from the Ministry of Education, Culture, Sports, Science, and Technology (16390073, 16390247, 80313237, 17023018, 17790182), YASUDA Medical Research Foundation, and the Kao Foundation for Arts and Sciences.

\section{References}

1 Ruotsalainen V, Ljungberg $\mathrm{P}$, Wartiovaara J, et al. Nephrin is specifically located at the slit diaphragm of glomerular podocytes. Proc Natl Acad Sci USA 1999;96:7962-7967.

2 Kestila M, Lenkkeri U, Mannikko M, et al. Positionally cloned gene for a novel glomerular protein-nephrinis mutated in congenital nephrotic syndrome. Mol Cell 1998;1:575-582.

3 Khoshnoodi J, Sigmundsson K, Ofverstedt LG, et al. Nephrin promotes cell-cell adhesion through homophilic interactions. Am J Pathol 2003;163:2337-2346.

4 Boute N, Gribouval O, Roselli S, et al. NPHS2, encoding the glomerular protein podocin, is mutated in autosomal recessive steroid-resistant nephrotic syndrome. Nat Genet 2000;24:349-354.

5 Schwarz K, Simons M, Reiser J, et al. Podocin, a raftassociated component of the glomerular slit diaphragm, interacts with CD2AP and nephrin. J Clin Invest 2001;108:1621-1629.

6 Dustin ML, Olszowy MW, Holdorf AD, et al. A novel adaptor protein orchestrates receptor patterning and cytoskeletal polarity in T-cell contacts. Cell 1998;94: 667-677.
7 Shih NY, Li J, Cotran R, et al. CD2AP localizes to the slit diaphragm and binds to nephrin via a novel C-terminal domain. Am J Pathol 2001;159:2303-2308.

8 Lehtonen S, Zhao F, Lehtonen E. CD2-associated protein directly interacts with the actin cytoskeleton. Am J Physiol Renal Physiol 2002;283:F734-F743.

9 Shih NY, Li J, Karpitskii V, et al. Congenital nephrotic syndrome in mice lacking CD2-associated protein. Science 1999;286:312-315.

$10 \mathrm{Kim}$ JM, Wu H, Green G, et al. CD2-associated protein haploinsufficiency is linked to glomerular disease susceptibility. Science 2003;300:1298-1300.

11 Donoviel DB, Freed DD, Vogel H, et al. Proteinuria and perinatal lethality in mice lacking NEPH1, a novel protein with homology to NEPHRIN. Mol Cell Biol 2001;21:4829-4836.

12 Barletta GM, Kovari IA, Verma RK, et al. Nephrin and Neph1 co-localize at the podocyte foot process intercellular junction and form cis hetero-oligomers. J Biol Chem 2003;278:19266-19271.

13 Liu G, Kaw B, Kurfis J, et al. Neph1 and nephrin interaction in the slit diaphragm is an important determinant of glomerular permeability. J Clin Invest 2003;112:209-221.

14 Kaplan JM, Kim SH, North KN, et al. Mutations in ACTN4, encoding alpha-actinin-4, cause familial focal segmental glomerulosclerosis. Nat Genet 2000;24: 251-256.

15 Huber TB, Kottgen M, Schilling B, et al. Interaction with podocin facilitates nephrin signaling. J Biol Chem 2001;276:41543-41546.

16 Sellin L, Huber TB, Gerke P, et al. NEPH1 defines a novel family of podocin interacting proteins. FASEB J 2003;17:115-117.

17 Roselli S, Heidet L, Sich M, et al. Early glomerular filtration defect and severe renal disease in podocindeficient mice. Mol Cell Biol 2004;24:550-560.

18 Stevenson BR, Siliciano JD, Mooseker MS, et al. Identification of ZO-1: a high molecular weight polypeptide associated with the tight junction (zonula occludens) in a variety of epithelia. J Cell Biol 1986;103:755-766.

19 Kurihara H, Anderson JM, Farquhar MG. Diversity among tight junctions in rat kidney: glomerular slit diaphragms and endothelial junctions express only one isoform of the tight junction protein ZO-1. Proc Natl Acad Sci USA 1992;89:7075-7079.

20 Schnabel E, Anderson JM, Farquhar MG. The tight junction protein ZO-1 is concentrated along slit diaphragms of the glomerular epithelium. J Cell Biol 1990;111:1255-1263.

21 Huber TB, Schmidts M, Gerke P, et al. The carboxyl terminus of Neph family members binds to the PDZ domain protein zonula occludens-1. J Biol Chem 2003; 278:13417-13421.

22 Dobrosotskaya I, Guy RK, James GL. MAGI-1, a membrane-associated guanylate kinase with a unique arrangement of protein-protein interaction domains. J Biol Chem 1997;272:31589-31597.

23 Ide N, Hata Y, Nishioka $\mathrm{H}$, et al. Localization of membrane-associated guanylate kinase (MAGI)-1/BAIassociated protein (BAP) 1 at tight junctions of epithelial cells. Oncogene 1999;18:7810-7815.

24 Patrie KM, Drescher AJ, Goyal M, et al. The membraneassociated guanylate kinase protein MAGI-1 binds megalin and is present in glomerular podocytes. J Am Soc Nephrol 2001;12:667-677. 
25 Hirabayashi S, Tajima M, Yao I, et al. JAM4, a junctional cell adhesion molecule interacting with a tight junction protein, MAGI-1. Mol Cell Biol 2003; 23:4267-4282.

26 Patrie KM, Drescher AJ, Welihinda A, et al. Interaction of two actin-binding proteins, synaptopodin and alpha-actinin-4, with the tight junction protein MAGI-1. J Biol Chem 2002;277:30183-30190.

27 Tajima M, Hirabayashi S, Yao I, et al. Roles of immunoglobulin-like loops of junctional cell adhesion molecule 4; involvement in the subcellular localization and the cell adhesion. Genes Cells 2003;8:759-768.

28 Yuan H, Takeuchi E, Taylor GA, et al. Nephrin dissociates from actin, and its expression is reduced in early experimental membranous nephropathy. J Am Soc Nephrol 2002;13:946-956.

29 Kawachi H, Koike H, Kurihara H, et al. Cloning of rat homologue of podocin: expression in proteinuric states and in developing glomeruli. J Am Soc Nephrol 2003;14:46-56.

30 Nishimura W, Iizuka T, Hirabayashi S, et al. Localization of BAI-associated protein $1 /$ membrane-associated guanylate kinase- 1 at adherens junctions in normal rat kidney cells: polarized targeting mediated by the carboxyl-terminal PDZ domains. J Cell Physiol 2000;185:358-365.

31 Ohno H, Hirabayashi S, Kansaku A, et al. Carom: a novel membrane-associated guanylate kinase-interacting protein with two SH3 domains. Oncogene 2003;22: 8422-8431.

32 Orikasa M, Matsui K, Oite T, et al. Massive proteinuria induced in rats by a single intravenous injection of a monoclonal antibody. J Immunol 1988;141:807-814.

33 Kawachi H, Koike H, Kurihara H, et al. Cloning of rat nephrin: expression in developing glomeruli and in proteinuric states. Kidney Int 2000;57:1949-1961.

34 Topham PS, Kawachi H, Haydar SA, et al. Nephritogenic mAb 5-1-6 is directed at the extracellular domain of rat nephrin. J Clin Invest 1999;104:1559-1566.

35 Miettinen A, Dekan G, Farquhar MG. Monoclonal antibodies against membrane proteins of the rat glomerulus. Immunochemical specificity and immunofluorescence distribution of the antigens. Am J Pathol 1990;137:929-944.

36 Ichimura K, Kurihara H, Sakai T. Actin filament organization of foot processes in rat podocytes. J Histochem Cytochem 2003;51:1589-1600.

37 Songyang Z, Fanning AS, Fu C, et al. Recognition of unique carboxyl-terminal motifs by distinct PDZ domains. Science 1997;275:73-77.

38 Yuan H, Takeuchi E, Salant DJ. Podocyte slit-diaphragm protein nephrin is linked to the actin cytoskeleton. Am J Physiol Renal Physiol 2002;282: F585-F591.

39 Kawachi H, Abrahamson DR, St John PL, et al. Developmental expression of the nephritogenic antigen of monoclonal antibody 5-1-6. Am J Pathol 1995;147:823-833.

40 Guan N, Ding J, Deng J, et al. Key molecular events in puromycin aminonucleoside nephrosis rats. Pathol Int 2004;54:703-711.

41 Rodewald R, Karnovsky MJ. Porous substructure of the glomerular slit diaphragm in the rat and mouse. J Cell Biol 1974;60:423-433.

42 Reiser J, Kriz W, Kretzler M, et al. The glomerular slit diaphragm is a modified adherens junction. J Am Soc Nephrol 2000;11:1-8.
43 Inoue $\mathrm{T}$, Yaoita $\mathrm{E}$, Kurihara $\mathrm{H}$, et al. FAT is a component of glomerular slit diaphragms. Kidney Int 2001;59:1003-1012.

44 Lehtonen S, Lehtonen E, Kudlicka K, et al. Nephrin forms a complex with adherens junction proteins and CASK in podocytes and in Madin-Darby canine kidney cells expressing nephrin. Am J Pathol 2004;165: 923-936.

45 Huber TB, Kottgen M, Schilling B, et al. Interaction with podocin facilitates nephrin signaling. J Biol Chem 2001;276:41543-41546.

46 Huber TB, Hartleben B, Kim J, et al. Nephrin and CD2AP associate with phosphoinositide $3-\mathrm{OH}$ kinase and stimulate AKT-dependent signaling. Mol Cell Biol 2003;23:4917-4928.

47 Hung AY, Sheng M. PDZ domains: structural modules for protein complex assembly. J Biol Chem 2002;277: 5699-5702.

48 Feng W, Shi Y, Li M, et al. Tandem PDZ repeats in glutamate receptor-interacting proteins have a novel mode of PDZ domain-mediated target binding. Nat Struct Biol 2003;10:972-978.

49 Lehtonen S, Ryan JJ, Kudlicka K, et al. Cell junctionassociated proteins IQGAP1, MAGI-2, CASK, spectrins, and a-actinin are components of the nephrin multiprotein complex. Proc Natl Acad Sci USA 2005; 102:9814-9819.

50 Hirao K, Hata $\mathrm{Y}$, Ide $\mathrm{N}$, et al. A novel multiple PDZ domain-containing molecule interacting with $\mathrm{N}$ methyl-D-aspartate receptors and neuronal cell adhesion proteins. J Biol Chem 1998;273:21105-21110.

51 Tsukita S, Furuse M, Itoh M. Multifunctional strands in tight junctions. Nat Rev Mol Cell Biol 2001;2: 285-293.

52 Hurd TW, Gao L, Roh $\mathrm{MH}$, et al. Direct interaction of two polarity complexes implicated in epithelial tight junction assembly. Nat Cell Biol 2003; 5:137-142.

53 Schnabel E, Dekan G, Miettinen A, et al. Biogenesis of podocalyxin-the major glomerular sialoglycoprotein-in the newborn rat kidney. Eur J Cell Biol 1989;48:313-326.

54 Kurihara H, Anderson JM, Kerjaschki D, et al. The altered glomerular filtration slits seen in puromycin aminonucleoside nephrosis and protamine sulfatetreated rats contain the tight junction protein ZO-1. Am J Pathol 1992;141:805-816.

55 Ruotsalainen V, Patrakka J, Tissari P, et al. Role of nephrin in cell junction formation in human nephrogenesis. Am J Pathol 2000;157:1905-1916.

56 Verma R, Wharram B, Kovari I, et al. Fyn binds to and phosphorylates the kidney slit diaphragm component Nephrin. J Biol Chem 2003;278:20716-20723.

57 Mino A, Ohtsuka $\mathrm{T}$, Inoue $\mathrm{E}$, et al. Membraneassociated guanylate kinase with inverted orientation (MAGI)-1/brain angiogenesis inhibitor 1-associated protein (BAP1) as a scaffolding molecule for Rap small $\mathrm{G}$ protein GDP/GTP exchange protein at tight junctions. Genes Cells 2000;5:1009-1016.

58 Dobrosotskaya IY, James GL. MAGI-1 interacts with beta-catenin and is associated with cell-cell adhesion structures. Biochem Biophys Res Commun 2000;270: 903-909.

59 Dobrosotskaya IY. Identification of mNET1 as a candidate ligand for the first PDZ domain of MAGI-1. Biochem Biophys Res Commun 2001;283: 969-975. 